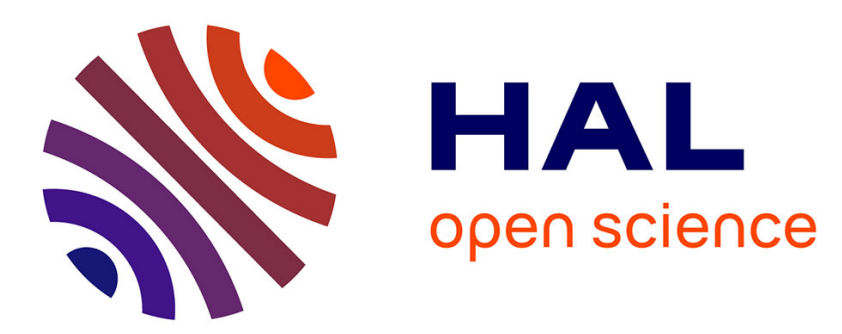

\title{
Three-dimensional instabilities and transient growth of a counter-rotating vortex pair
}

Claire Donnadieu, Sabine Ortiz, Jean-Marc Chomaz, Paul Billant

\section{To cite this version:}

Claire Donnadieu, Sabine Ortiz, Jean-Marc Chomaz, Paul Billant. Three-dimensional instabilities and transient growth of a counter-rotating vortex pair. Physics of Fluids, 2009, 21 (9), pp.094102. 10.1063/1.3220173 . hal-01025605

\section{HAL Id: hal-01025605 \\ https://hal-polytechnique.archives-ouvertes.fr/hal-01025605}

Submitted on 18 Jul 2014

HAL is a multi-disciplinary open access archive for the deposit and dissemination of scientific research documents, whether they are published or not. The documents may come from teaching and research institutions in France or abroad, or from public or private research centers.
L'archive ouverte pluridisciplinaire HAL, est destinée au dépôt et à la diffusion de documents scientifiques de niveau recherche, publiés ou non, émanant des établissements d'enseignement et de recherche français ou étrangers, des laboratoires publics ou privés. 


\section{AIP | Physics of Fluids}

\section{Three-dimensional instabilities and transient growth of a counter-rotating vortex pair}

Claire Donnadieu, Sabine Ortiz, Jean-Marc Chomaz, and Paul Billant

Citation: Physics of Fluids (1994-present) 21, 094102 (2009); doi: 10.1063/1.3220173

View online: http://dx.doi.org/10.1063/1.3220173

View Table of Contents: http://scitation.aip.org/content/aip/journal/pof2/21/9?ver=pdfcov

Published by the AIP Publishing

\section{Articles you may be interested in}

Three-dimensional Lagrangian transport phenomena in unsteady laminar flows driven by a rotating sphere Phys. Fluids 25, 093602 (2013); 10.1063/1.4819901

Two-dimensional viscous flow simulation of a shock accelerated heavy gas cylinder Phys. Fluids 23, 024102 (2011); 10.1063/1.3553282

Analytical and numerical investigations of laminar and turbulent Poiseuille-Ekman flow at different rotation rates Phys. Fluids 22, 105104 (2010); 10.1063/1.3488039

On the development of three-dimensional vortex breakdown in cylindrical regions

Phys. Fluids 18, 084105 (2006); 10.1063/1.2338065

Widnall instabilities in vortex pairs

Phys. Fluids 15, 1861 (2003); 10.1063/1.1575752

\section{A|P| $\left.\right|_{\text {Applied Physics }} ^{\text {Journal of }}$}




\title{
Three-dimensional instabilities and transient growth of a counter-rotating vortex pair
}

\author{
Claire Donnadieu, ${ }^{1}$ Sabine Ortiz, ${ }^{1,2}$ Jean-Marc Chomaz, ${ }^{1}$ and Paul Billant ${ }^{1}$ \\ ${ }^{1}$ LadHyX, CNRS-Ecole Polytechnique, F-91128 Palaiseau Cedex, France \\ ${ }^{2}$ UME/DFA, ENSTA, Chemin de la Hunière, 91761 Palaiseau Cedex, France
}

(Received 4 February 2009; accepted 1 July 2009; published online 15 September 2009)

\begin{abstract}
This paper investigates the three-dimensional instabilities and the transient growth of perturbations on a counter-rotating vortex pair. The two dimensional base flow is obtained by a direct numerical simulation initialized by two Lamb-Oseen vortices that quickly adjust to a flow with elliptic vortices. In the present study, the Reynolds number, $\operatorname{Re}_{\Gamma}=\Gamma / \nu$, with $\Gamma$ the circulation of one vortex and $\nu$ the kinematic viscosity, is taken large enough for the quasi steady assumption to be valid. Both the direct linearized Navier-Stokes equation and its adjoint are solved numerically and used to investigate transient and long time dynamics. The transient dynamics is led by different regions of the flow, depending on the optimal time considered. At very short times compared to the advection time of the dipole, the dynamics is concentrated on the points of maximal strain of the base flow, located at the periphery of the vortex core. At intermediate times, depending on the symmetry of the perturbation, one of the hyperbolic stagnation points provides the optimal amplification by stretching of the perturbation vorticity as in the classical hyperbolic instability. The growth of both short time and intermediate time transient perturbations are non- or weakly dependent of the axial wavenumber whereas the long time behavior strongly selects narrow bands of wavenumbers. We show that, for all unstable spanwise wavenumbers, the transient dynamics last until the nondimensional time $t=2$, during which the dipole has traveled twice the separation distance between vortices $b$. During that time, all the wavenumbers exhibit a transient growth of energy by a factor of 50, for the Reynolds number $\operatorname{Re}_{\Gamma}=2000$. For time larger than $t=2$, energy starts growing at a rate given by the standard temporal stability theory. For all wavenumbers and two Reynolds numbers, $\operatorname{Re}_{\Gamma}=2000$ and $\operatorname{Re}_{\Gamma}=10^{5}$, different instability branches have been computed using a high resolution Krylov method. At large Reynolds number, the computed Crow and elliptic instability branches are in excellent agreement with the inviscid theory [S. C. Crow, AIAA J. 8, 2172 (1970); S. Le Dizes and F. Laporte, J. Fluid Mech. 471, 120 (2002)] and numerical analysis [D. Sipp and L. Jacquin, Phys. Fluids 15, 1861 (2003)]. A novel oscillatory elliptic instability involving Kelvin waves with azimuthal wavenumbers $m=0$ and $|m|=2$, that was missed in previous numerical analysis [D. Sipp and L. Jacquin, Phys. Fluids 15, 1861 (2003)] is found. For the stationary elliptic instability, we show that viscous effect may be estimated using the large Reynolds number direct and adjoint eigenmodes. This asymptotically exact estimate of the viscous damping of elliptic instability mode agrees with our direct numerical computation of instability branches at moderate Reynolds number and demonstrates that formula proposed by Le Dizes and Laporte [J. Fluid Mech. 471, 120 (2002)] strongly over estimated the viscous correction. (C) 2009 American Institute of Physics. [doi:10.1063/1.3220173]
\end{abstract}

\section{INTRODUCTION}

Trailing vortices behind aircrafts consist of a horizontal pair of counter-rotating vortices propagating downwards. Such a dipole can be hazardous to following aircrafts during the take-off and landing since it can persist over a long time and can induce a strong rolling moment to following aircrafts. Safety regulation imposes a minimum distance between airplanes to avoid such danger.

The dynamics of counter-rotating vortices has been widely studied. These studies have shown that a pair of counter-rotating vortices is unstable with respect to threedimensional perturbations. A long- and a short-wavelength instabilities have been observed and numerically analyzed. This confirmed the theoretical work of $\mathrm{Crow}^{1}$ on a pair of vortex filaments predicting the existence of a longwavelength symmetric (with respect to the plane separating the two vortices) instability with a wavelength of about five to ten times the vortex core separation distance. The existence of a short-wavelength elliptic instability has been described theoretically by Moore and Saffman ${ }^{2}$ and Tsai and Widnall, ${ }^{3}$ who have investigated the stability of a vortex patch in a uniform strain field. They have shown that this instability originates from the resonant interaction between the strain and Kelvin waves of azimuthal wavenumbers $m$ $=1$ and $m=-1$ when both waves have the same frequency $\omega$ and is particularly intense for $\omega=0$. Later, Pierrehumbert ${ }^{4}$ showed that an unbounded strained vortex, with elliptic streamlines, is unstable to three-dimensional instabilities. In this unbounded limit, Bayly ${ }^{5}$ and Waleffe, ${ }^{6}$ using a local ap- 
proach, have shown that the elliptic instability appears as a parametric instability of inertial waves of zero frequency in the fixed frame and is therefore similar to the one discovered by Tsai and Widnall. ${ }^{3}$ Numerous papers ever since have focused on this instability with both numerical and theoretical studies (Sipp and Jacquin, ${ }^{7}$ Billant et al., ${ }^{8}$ Laporte and Corjon, ${ }^{9}$ Le Dizès and Laporte, ${ }^{10}$ see the review by Kerswell $^{11}$ ). The general case of the resonant interaction between Kelvin waves of azimuthal wavenumbers $m$ and $m^{\prime}=m-2$ has been also analyzed (Tsai and Widnall, ${ }^{3}$ Eloy and Le Dizès ${ }^{12}$ ). Effect of axial flow and density variations in the core of the vortices have been discussed in Mayer and Powell, ${ }^{13}$ Lacaze et al.,${ }^{14}$ Coquart et al. ${ }^{15}$ among others. In particular axial flow on a single vortex is known to be strongly destabilizing and to promote helical instability through a generalized centrifugal instability (Leibovich and Stewartson ${ }^{16}$ ). Stability and transient growth on such a swirling flow have recently been addressed (Ben-Dov et al., ${ }^{17}$ Heaton and Peake ${ }^{18}$ ) but will not be discussed further since no axial flow will be considered in the present paper.

A few experimental works have described the shortwavelength instability experienced by a pair of counterrotating vortices. Among them, Leweke and Williamson ${ }^{19}$ observed the internal deformation of the vortex cores characteristic of the elliptic instability and have been able to obtain quantitative measurements of the wavelength and the growth rate in agreement with theoretical analysis. In their laboratory experiments, they have observed preferentially antisymmetric deformations of the cores. This selectivity is not predicted by linear stability of inviscid base state, since in that case, both symmetric and antisymmetric elliptic modes present nearly equal growth rate (Sipp and Jacquin, ${ }^{7}$ Billant et $a l^{8}{ }^{8}$. Sipp and Jacquin ${ }^{7}$ showed, by taking into account the unsteadiness of the flow due to slow viscous diffusion and using quasisteady approximation with a shape assumption, a growth of the antisymmetric mode stronger than that of the symmetric one, which may account for the observed selection. The present paper will address the possibility of transient growth on such a flow. In particular we will show that the antisymmetric mode also presents a higher sensitivity to initial perturbations than the symmetric mode.

In this paper, a three-dimensional stability analysis of a Lamb-Oseen vortex pair is performed in Sec. II. Transient growths of perturbations on the counter-rotating vortex pair are studied in Sec. III. In Sec. III A, we give the adjoint equations and discuss the adjoint eigenmodes. After the study of the large time energy growth of the perturbations in Sec. III B, we focus in Sec. III C on the short time behavior of the perturbations on the dipole by computing the optimal linear perturbations with a direct-adjoint technique similar to the one introduced by Luchini. ${ }^{20}$ Recent related works, in particular, by Antkowiak and Brancher, ${ }^{21}$ Pradeep and Hussain, ${ }^{22}$ and Brion, Sipp and Jacquin ${ }^{23}$ on optimal amplification and transient growth on a single vortex or on a pair of vortices are discussed in the concluding section.

\section{LINEAR THREE-DIMENSIONAL STABILITY ANALYSIS OF A VORTEX PAIR}

We investigate the three-dimensional instabilities of a horizontal pair of counter-rotating vortices of initial circulation $\Gamma_{0}$, vortex radius $a_{0}$ and vortex separation $b_{0}$. The spatial coordinates are Cartesian $(x, y, z)$, corresponding, respectively, to transverse, axial and vertical directions. The vortex pair propagates downward along the vertical direction with the initial advection velocity $W_{0}=\Gamma_{0} / 2 \pi b_{0}$. The initial state is the superposition of two circular Lamb-Oseen vortices with two-dimensional initial vorticity field $\omega_{B y}$ given by

$$
\begin{aligned}
\omega_{B y}(x, z, t=0)= & \frac{\Gamma_{0}}{\pi a_{0}^{2}} e^{-\left(x-x_{1}\right)^{2}+\left(z-z_{1}\right)^{2} / a_{0}^{2}} \\
& -\frac{\Gamma_{0}}{\pi a_{0}^{2}} e^{-\left(x-x_{2}\right)^{2}+\left(z-z_{2}\right)^{2} / a_{0}^{2}},
\end{aligned}
$$

where $\left(x_{1}, z_{1}\right)$ and $\left(x_{2}=x_{1}+b_{0}, z_{2}=z_{1}\right)$ are the initial coordinates of the two vortex centroids.

\section{A. 2D base state}

The base state is computed from this initial state by a $2 \mathrm{D}$ numerical simulation described in Appendix A. As described by Sipp et al. ${ }^{24}$ the counter-rotating vortices adapt to each other by a two-dimensional process in two steps. First, the mutual strain imposed on one vortex by the other drives the vortices to become elliptical. An equilibrium is rapidly reached and a quasisteady solution of the Euler equations is established. Then, the dipole belongs to a unique family characterized by its aspect ratio $a / b$ (Sipp et $a .^{24}$ ) for which $\Gamma$ and $b$ are constant and $a$ evolves by viscous diffusion according to the law (Batchelor ${ }^{25}$ ): $a^{2}=a_{0}^{2}+4 \nu t$, where $\nu$ is the viscosity of the flow. At that stage, for large Reynolds numbers, the evolution of the dipole in the frame moving with the vortices at the vertical velocity $W_{0}$ is therefore extremely slow and, at each instant, we can perform a quasistatic stability analysis by freezing the instantaneous flow field.

Figure 1(a) represents the isovalues of the axial vorticity obtained in the diffusive regime at time $t \Gamma_{0} / 2 \pi b_{0}^{2}=3$ for $\operatorname{Re}_{\Gamma_{0}}=\Gamma_{0} / \nu=2400$. The aspect ratio of the dipole has evolved from its initial value $a_{0} / b_{0}=0.1$ to the value $a / b=0.206$, where the vortex radius $a$ is computed using the vorticity polar moment: $a^{2}=\left\langle\left[\left(x-x_{2}\right)^{2}+\left(z-z_{2}\right)^{2}\right] \omega_{B y}\right\rangle /\left\langle\omega_{B y}\right\rangle$ with $\langle$. denoting the integration over the semi-infinite domain $x>0$ and the distance between the two vortices is $b=\left|x_{2}-x_{1}\right|$, $\left(x_{1}, z_{1}\right)$, and $\left(x_{2}, z_{2}\right)$ being the location of the vorticity extrema. This base state is symmetric with respect to the axis $x=0$,

$$
\begin{aligned}
& {\left[u_{B}, 0, w_{B}\right](x, z)=\left[-u_{B}, 0, w_{B}\right](-x, z),} \\
& {\left[0, \omega_{B y}, 0\right](x, z)=\left[0,-\omega_{B y}, 0\right](-x, z),}
\end{aligned}
$$

where $u_{B}$ and $w_{B}$ are, respectively, the transverse and the vertical velocity of the base state. 
(a)

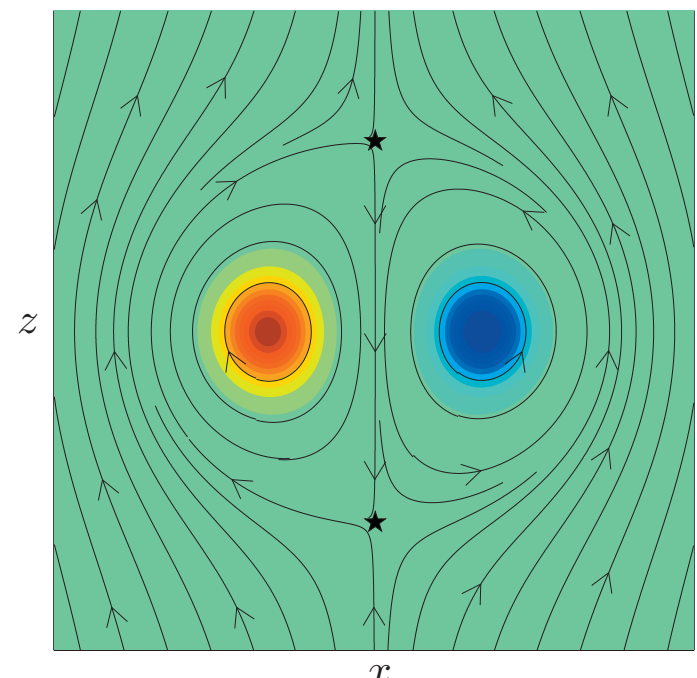

(b)

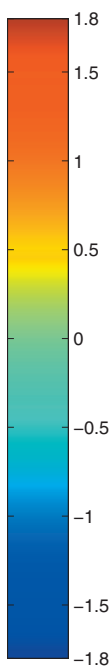

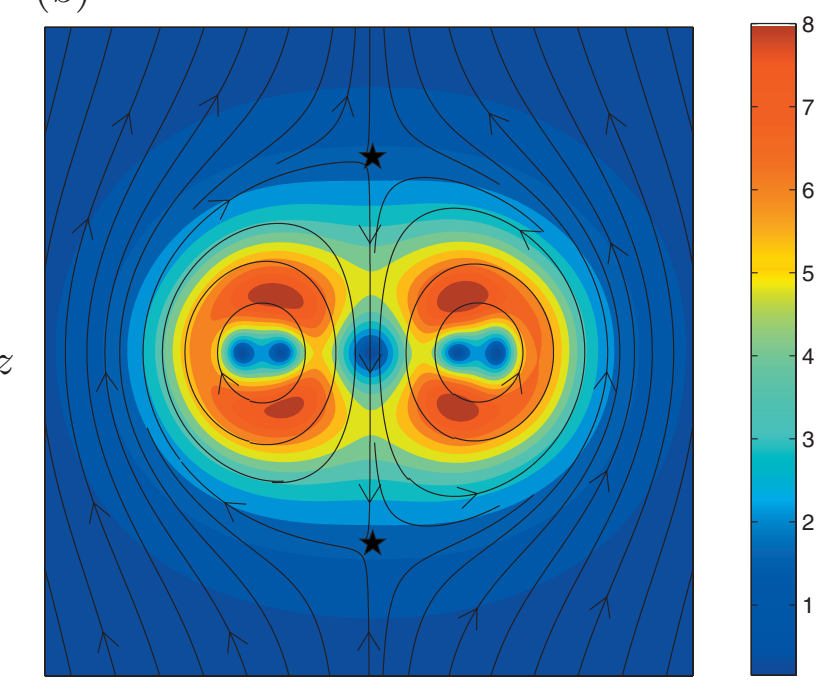

$\mathscr{X}$

FIG. 1. (Color) Isovalues of (a) the axial vorticity of the base state $\omega_{B y} 2 \pi a^{2} / \Gamma$ and (b) the local strain $\epsilon 2 \pi b^{2} / \Gamma$ in the ( $\left.x, z\right)$ plane for $a / b=0.206$ and $\operatorname{Re}_{\Gamma_{0}}=\Gamma_{0} / \nu=2400$. The stars represent the two hyperbolic points of the base state and the arrowed lines sketch the streamlines of the base state. The size of the domain shown is $3 b \times 3 b$ where $b$ is the separation distance between the two vortex centers.

Figure 1(a) shows the streamlines in the frame moving with the dipole. The base state streamlines possess two hyperbolic points [indicated by stars on Figs. 1(a) and 1(b)]. Figure 1(b) represents the strain

$$
\epsilon(x, z)=\sqrt{\left(\frac{\partial w_{B}}{\partial z}\right)^{2}+\frac{1}{4}\left(\frac{\partial u_{B}}{\partial z}+\frac{\partial w_{B}}{\partial x}\right)^{2}}
$$

of the base state. The strain is maximum at the periphery of the vortex cores [dark red in Fig. 1(b)] in nontrivial locations.

\section{B. Linearized equations}

For the stability analysis, the base state presented in Fig. 1 is frozen in the frame moving with the dipole. This frame of reference is taken by subtracting the advection velocity of the dipole to the base state velocity field. The quasistatic stability analysis is valid as long as the time scale of the instability is smaller than the diffusion time scale $T_{\nu}=a^{2} / \nu$. As known from previous studies, the mutual strain exerted by one vortex on the other is the driving instability mechanism for both Crow and elliptic instabilities, and the time scale of the instability is of the order $T_{i}=2 \pi b^{2} / \Gamma$. We have, $T_{\nu} / T_{i}=\operatorname{Re}(a / b)^{2}$ where $\operatorname{Re}=W b / \nu$ is the Reynolds number, with $W=\Gamma / 2 \pi b$ the advection velocity of the dipole. The quasistatic approximation implies $T_{i} \ll T_{\nu}$, i.e., $\operatorname{Re}(a / b)^{2} \gg 1$, in the following, we will use the circulation Reynolds number $\operatorname{Re}_{\Gamma}=2 \pi \operatorname{Re}=\Gamma / \nu$. This condition is always fulfilled in this paper, for $\operatorname{Re}_{\Gamma}=2000, T_{i}=4$, and $T_{\nu}=51$, for $\operatorname{Re}_{\Gamma}=10^{5}, T_{i}=4$, and $T_{\nu}=2546$ meaning that for $\operatorname{Re}_{\Gamma}=2000$, $T_{i} / T_{\nu}=(\pi / 4) 10^{-1}$ and for $\operatorname{Re}_{\Gamma}=10^{5}, T_{i} / T_{\nu}=(\pi / 2) 10^{-3}$. The procedure is here identical to that of Sipp and Jacquin ${ }^{7}$ except that we will carry out a finite Reynolds number viscous stability analysis.

Infinitesimal three-dimensional perturbations are superposed to the frozen base state,

$$
\begin{aligned}
& \mathbf{u}^{\prime}(x, y, z, t)=\mathbf{u}_{B}(x, z)+\mathbf{u}(x, y, z, t), \\
& \boldsymbol{\omega}^{\prime}(x, y, z, t)=\boldsymbol{\omega}_{B}(x, z)+\boldsymbol{\omega}(x, y, z, t), \\
& p^{\prime}(x, y, z, t)=p_{B}(x, z)+p(x, y, z, t),
\end{aligned}
$$

where $[\mathbf{u}, \boldsymbol{\omega}, p](x, y, z, t)$, the velocity, the vorticity, and the pressure of the three-dimensional perturbation are solutions of the linearized Navier-Stokes equations,

$$
\begin{aligned}
& \frac{\partial \mathbf{u}}{\partial t}=\mathbf{u}_{B} \times \boldsymbol{\omega}+\mathbf{u} \times \boldsymbol{\omega}_{B}-\nabla\left(p+\mathbf{u} \cdot \mathbf{u}_{B}\right)+\nu \Delta \mathbf{u}, \\
& \nabla \cdot \mathbf{u}=0,
\end{aligned}
$$

where $p$ is the pressure normalized by the constant density.

As the base state is uniform along the $y$ axis, the perturbations can be decomposed into normal modes,

$$
[\mathbf{u}, \boldsymbol{\omega}, p](x, y, z, t)=[\widetilde{\mathbf{u}}, \widetilde{\boldsymbol{\omega}}, \widetilde{p}](x, z, t) \mathrm{e}^{i k_{y} y}+\text { c.c. },
$$

where $k_{y}$ is the axial wavenumber and c.c. denotes the complex conjugate.

\section{Numerical method}

The linearized Navier-Stokes equation (5) are integrated using the pseudospectral method in Cartesian coordinates with periodic boundary conditions described in Delbende et al. ${ }^{26}$ The velocity, vorticity, and pressure perturbations are expressed in Fourier space by application of the Fourier transform:

$$
[\widetilde{\mathbf{u}}, \widetilde{\boldsymbol{\omega}}, \widetilde{p}](x, z, t)=\iint[\hat{\mathbf{u}}, \hat{\boldsymbol{\omega}}, \hat{p}]\left(k_{x}, k_{z}, t\right) e^{i\left(k_{x} x+k_{z} z\right)} d k_{x} d k_{z} .
$$

In spectral space, the linear Navier-Stokes equation (5) becomes 


$$
\frac{\partial \hat{\mathbf{u}}}{\partial t}=\mathbf{P}(\mathbf{k})\left[\mathbf{u}_{B} \times \widehat{\boldsymbol{\omega}+\mathbf{u}} \times \boldsymbol{\omega}_{B}\right]-\nu \mathbf{k}^{2} \hat{\mathbf{u}},
$$

where $\mathbf{k}=\left(k_{x}, k_{y}, k_{z}\right)$ is the total wavevector and $\mathbf{P}(\mathbf{k})$ is the projection operator on the space of divergence-free fields which, in Fourier space, may be expressed as a tensor with components $P_{i j}=\delta_{i j}-k_{i} k_{j} / \mathbf{k}^{2}$. Introduction of this operator suppresses the term $\nabla\left(p+\mathbf{u} \cdot \mathbf{u}_{B}\right)$. The cross-product terms $\mathbf{u}_{B} \times \boldsymbol{\omega}+\mathbf{u} \times \boldsymbol{\omega}_{B}$ are evaluated in the physical space. Time integration is performed with a second-order AdamsBashforth scheme whereas the dissipative term $\nu \Delta \mathbf{u}$ is integrated exactly in the Fourier space.

The eigenmodes are computed independently for each axial wavenumber $k_{y}$. For $k_{y} a$ larger than 0.3 , the domain size in the $x$ and $z$ directions is half that used to compute the base state $\left(\mathbf{u}_{B}, \boldsymbol{\omega}_{B}\right)$ (see Appendix A), i.e., $L_{x}=L_{z}=3 b$ with a Cartesian grid of $256 \times 256$. The accuracy and the convergence of the results have been tested by taking a larger box size and a finer resolution (see Appendix B for details). For wavenumbers $k_{y} a$ smaller than 0.3 , the size of the computational domain has been kept the same as that of the base flow $L_{x}=L_{z}=6 b$ with $512 \times 512$ grid points. Indeed, away from the vortex dipole, the perturbation with an axial wavenumber $k_{y}$ decreases exponentially with an evanescent length scale proportional to $k_{y}^{-1}$. Assuming periodicity in the relatively small box $L_{x}=L_{z}=3 b$ has negligible effect when $k_{y} a$ is larger than 0.3 but affects the results for $k_{y} a<0.3$ whereas, down to $k_{y} a=0.1$, the size $L_{x}=L_{z}=6 b$ is sufficiently large. The time step is set to $\delta t=10^{-3}$, with $b=2$ and $\Gamma=2 \pi$. The threedimensional perturbation has been initialized either by divergence-free white noise or by an eigenmode computed previously for a slightly different axial wavenumber in order to speed up the time convergence.

A Krylov method similar to the one described in Edwards $\mathrm{et} \mathrm{al}^{27}$ is implemented in order to retrieve with a reasonable precision the three leading eigenmodes. After an initial integration over a time $T=70$ obtained from a simulation initialized by white noise, six perturbation velocity fields $\widetilde{\mathbf{u}}$ are saved, at six successive times separated by $\Delta T=10$ in order to construct an orthonormalized basis which spans a six dimension Krylov subspace. The eigenvalues of the evolution operator projected in this subspace ${ }^{28}$ are computed.

\section{Three-dimensional unstable modes}

Since the base state is symmetric versus $x \rightarrow-x$, the eigenmodes can be decomposed into symmetric (same symmetry as the base state)

$$
\begin{aligned}
& {\left[\widetilde{u}_{x}, \widetilde{u}_{y}, \widetilde{u}_{z}\right](x, z)=\left[-\widetilde{u}_{x}, \widetilde{u}_{y}, \widetilde{u}_{z}\right](-x, z),} \\
& {\left[\widetilde{\omega}_{x}, \widetilde{\omega}_{y}, \widetilde{\omega}_{z}\right](x, z)=\left[\widetilde{\omega}_{x},-\widetilde{\omega}_{y},-\widetilde{\omega}_{z}\right](-x, z)}
\end{aligned}
$$

and into an antisymmetric family (opposite symmetry to the base state)

$$
\begin{aligned}
& {\left[\widetilde{u}_{x}, \widetilde{u}_{y}, \widetilde{u}_{z}\right](x, z)=\left[\widetilde{u}_{x},-\widetilde{u}_{y},-\widetilde{u}_{z}\right](-x, z),} \\
& {\left[\widetilde{\omega}_{x}, \widetilde{\omega}_{y}, \widetilde{\omega}_{z}\right](x, z)=\left[-\widetilde{\omega}_{x}, \widetilde{\omega}_{y}, \widetilde{\omega}_{z}\right](-x, z) .}
\end{aligned}
$$

Symmetric and antisymmetric eigenmodes are calculated separately, the symmetries being imposed at each time step during the time evolution. This procedure improves the precision on the eigenvalues when their growth rate is small but is not essential since we have checked that eigenvalues and eigenmodes are similar by either imposing the symmetry at posteriori on the Krylov subspace if the integration is run without imposing the symmetry or by not imposing the symmetry but by increasing the dimension of the Krylov subspace to 12 in order to retrieve simultaneously the modes without assuming the symmetries of the modes [Eqs. (9) and (10)].

Figure 2 shows the real part of the dimensional growth rate $\sigma_{r}$ scaled by $2 \pi b^{2} / \Gamma$, the strain imposed by one vortex on the other, of symmetric and antisymmetric modes as function of the dimensional axial wavenumber $k_{y}$ scaled by the vortex radius $a$ for Reynolds numbers $\operatorname{Re}_{\Gamma}=10^{5}$ [Fig. 2(a)] and $\operatorname{Re}_{\Gamma}=2000$ [Fig. 2(b)].

The different instability bands corresponding to the classical Crow, elliptical instabilities, and to oscillatory modes are described below.

\section{Crow instability}

The first band on the left of Figs. 2(a) and 2(b), at small wavenumber between $k_{y} a=0$ and $k_{y} a=0.3$, corresponds to the long-wavelength Crow instability, with a maximum growth rate $\sigma_{r} 2 \pi b^{2} / \Gamma=0.74$ for $\operatorname{Re}_{\Gamma}=10^{5}$ and 0.73 for $\operatorname{Re}_{\Gamma}=2000$ at the wavenumber $k_{y} a=0.19$, corresponding to $k_{y} b=0.92$. This instability is symmetric, antisymmetric modes being all stable for $k_{y} a$ smaller than 0.5 for $\operatorname{Re}_{\Gamma}=10^{5}$ and 1.9 for $\operatorname{Re}_{\Gamma}=2000$. The dashed line in Fig. 2 represents the theoretical inviscid predictions of $\mathrm{Crow}^{1}$ for a pair of vortex filaments with an equivalent radius $a_{e}=1.36 a$, for long-wavelength disturbances, the coefficient 1.36 being derived from the bending mode dynamics taking into account the present Gaussian vorticity distribution $\left(\right.$ Widnall ${ }^{29}$ ). The predicted maximum growth rate at the wavenumber $k_{y} a=0.19$ is 0.79 which is in very good agreement with the numerical value. The axial vorticity $\widetilde{\omega}_{y}$ of the eigenmode (Fig. 3) at the maximum of the Crow instability band $k_{y} a=0.19$ is odd with respect to $x=0$ since the mode is symmetric.

This vorticity perturbation, when added to the base flow, induces a symmetric displacement of the base flow vortices along lines inclined at an angle of $45^{\circ}$ as predicted by the theory. ${ }^{1}$

\section{Elliptic instability}

For $\operatorname{Re}_{\Gamma}=10^{5}$, Fig. 2(a) shows three dominant branches of instability, with maximum nondimensional growth rate equal to 1.32 at wavenumber $k_{y} a=2.26,1.29$ at $k_{y} a=3.96$ and 1.24 at $k_{y} a=5.64$, which correspond to the elliptic instability and are well predicted by the inviscid theory of 
(a)

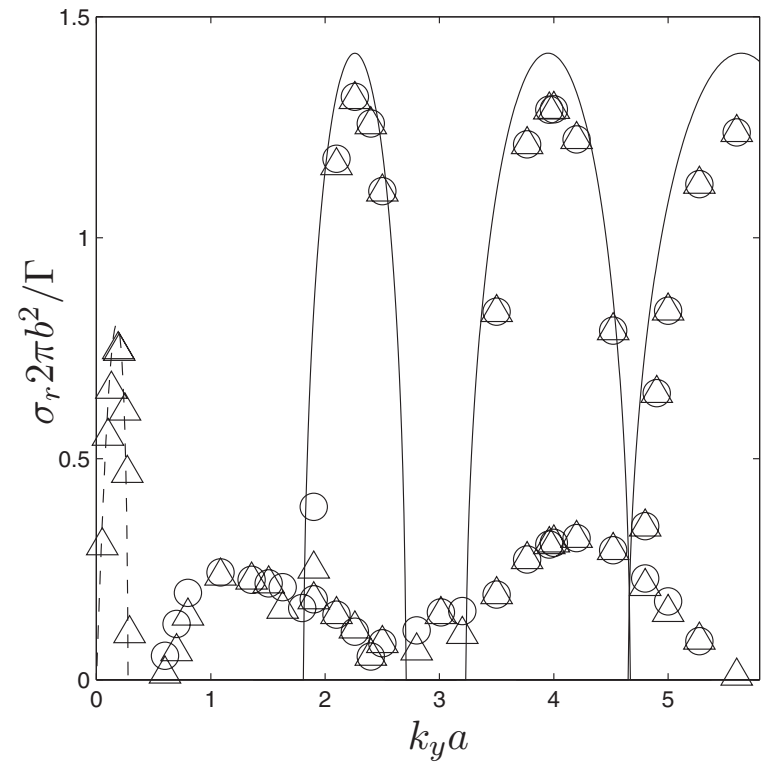

(b)

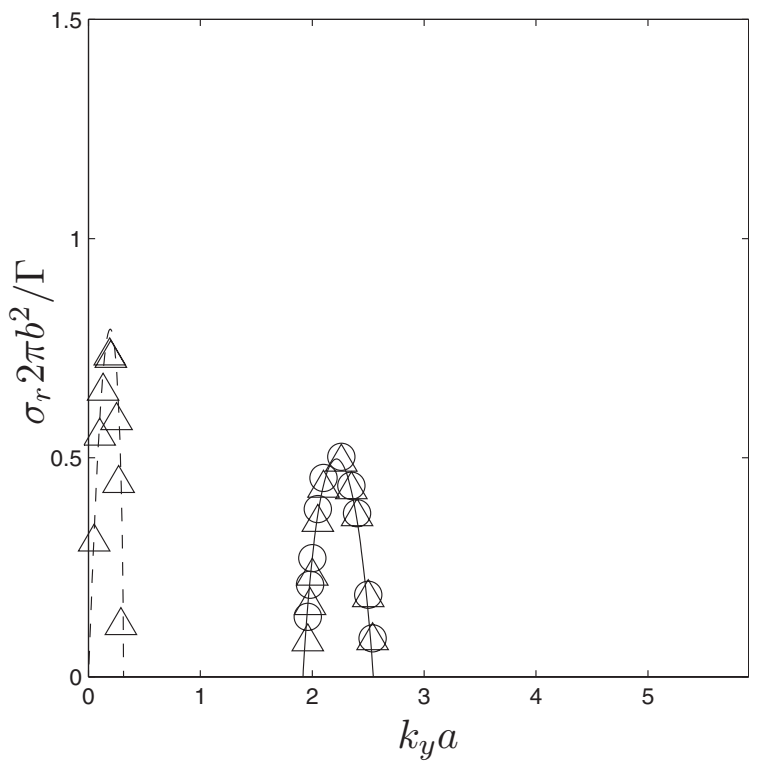

FIG. 2. Nondimensional growth rates $\sigma_{r} 2 \pi b^{2} / \Gamma$ of symmetric $(\triangle)$ and antisymmetric $(\bigcirc)$ modes as function of the nondimensional axial wavenumber $k_{y} a$ for (a) $\operatorname{Re}_{\Gamma}=10^{5}$ and (b) $\operatorname{Re}_{\Gamma}=2000$. Dashed line corresponds to the theory of Crow (Ref. 1) for a pair of vortex filaments. Continuous lines of (a) correspond to the inviscid theory of Le Dizès and Laporte (Ref. 10) for a pair of Lamb-Oseen vortices with a Gaussian vorticity profile in the inviscid limit and the continuous line of (b) is the present viscous prediction (C2), derived in Appendix C.

Le Dizès and Laporte. ${ }^{10,30}$ Each of these branches are double with family of symmetric and antisymmetric mode with almost identical growth rates, showing that the present value of $a / b=0.206$ is already small enough for the coupling between the elliptic instability that affect each vortex core to be negligible as assumed in Le Dizès and Laporte. ${ }^{10}$ The axial vorticity $\widetilde{\omega}_{y}$ of the eigenmode at the maximum of the two first instability branches $k_{y} a=2.26$ and $k_{y} a=3.96$ have been represented for both symmetries in Fig. 4.

For the low Reynolds number $\operatorname{Re}_{\Gamma}=2000$ shown in Fig. 2(b), only the first band with a maximum growth rate $\sigma_{r} 2 \pi b^{2} / \Gamma=0.5$ at wavenumber $k_{y} a=2.26$ is unstable. The present finite Reynolds number stability analysis shows that the viscous prediction proposed by Le Dizès and Laporte ${ }^{10}$ (not shown on the figure) is not valid since it predicts that this mode should be stable for $\operatorname{Re}_{\Gamma}=2000$ with a growth rate $\sigma_{r} 2 \pi b^{2} / \Gamma=-0.33$ at the maximum of the instability band. In Le Dizès and Laporte, ${ }^{10}$ the viscous damping is derived from the asymptotic formula of Landman and Saffman ${ }^{31}$ $\nu k_{y}^{2} / \cos ^{2} \xi$ valid at large $k_{y}$ for any inertial wave with a local wavevector $\mathbf{k}$, with a $k_{y}$ component along the rotation axis $y$ and making the angle $\xi$ with the $y$ axis. The angle $\xi$ is then obtained from the numerically computed frequency of the resonant Kelvin mode $m=1$ which, for the different branches, gives the fitted formula

$$
\cos \xi=\frac{1}{2}-\frac{(2.26+1.69 n)-k_{y} a}{14.8+9 n}
$$

where $n=0,1,2, \ldots$ is the index of the branch. The present direct stability analysis shows that such a procedure, probably legitimate for large axial wavenumbers, strongly overestimates (by nearly a factor of 2) the viscous damping of the first elliptic instability branches. Their viscous theory has not been displayed in Fig. 2(b). Instead, the solid curve reported in Fig. 2(b) is the present viscous prediction [Eq. (C2)] computed as a perturbation of the full evolution operator via a method presented in Appendix C. This new method takes into account the very spatial structure of the eigenmode by the use of the adjoint mode of the elliptic instability instead of assuming a plane wave expansion.

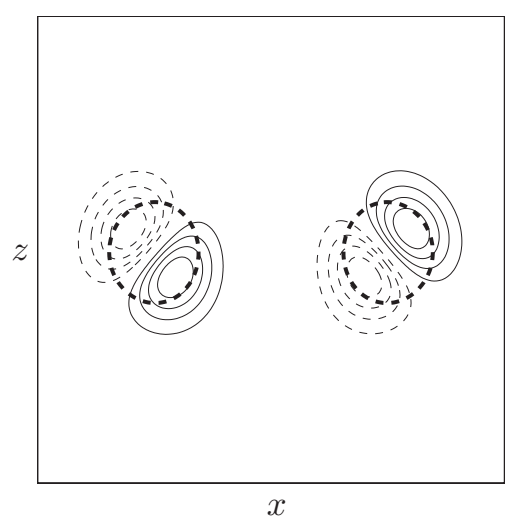

FIG. 3. Crow instability: Contours of the axial vorticity $\widetilde{\omega}_{y}$ of the eigenmode in the $(x, z)$ plane for $\operatorname{Re}_{\Gamma}=10^{5}$ at the leading wavenumber of the Crow instability branch: $k_{y} a=0.19$. The contour levels $\widetilde{\omega}_{y} /\left|\widetilde{\omega}_{y \max }\right|$ shown are $\pm 0.2, \pm 0.4, \pm 0.6$, and \pm 0.8 . Continuous lines correspond to positive vorticity and dashed lines correspond to negative vorticity. The heavy dashed lines correspond to the isocontours $\omega_{B y} /\left|\omega_{B y}\right|= \pm \exp (-1)$ of the base state. The size of the domain shown is $2 b \times 2 b$ whereas the computation domain is $6 b \times 6 b$. 
ANTISYMMETRIC

(a)

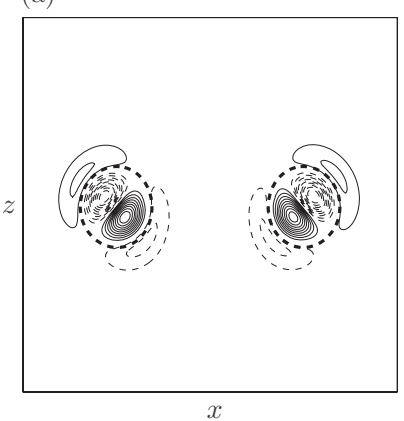

(c)

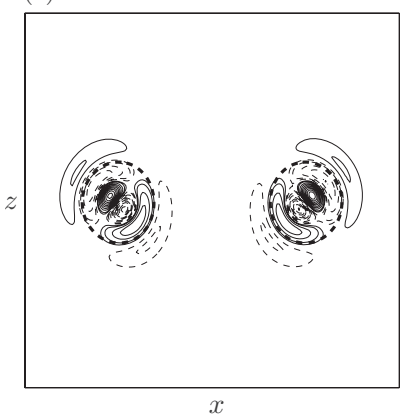

SYMMETRIC

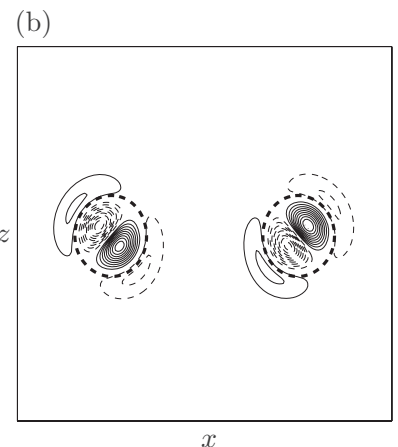

(d)

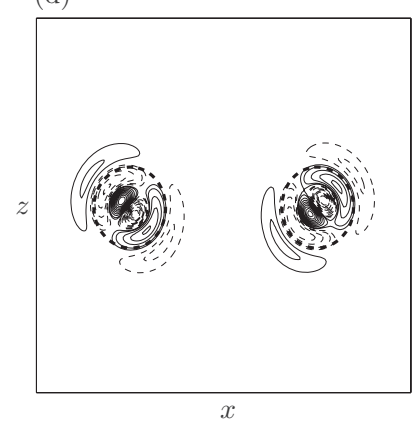

FIG. 4. Elliptic instability: Same as Fig. 3 but for the first band of the elliptic instability at $k_{y} a=2.26[(\mathrm{a})$ and (b)] and for the second band at $k_{y} a=3.96[$ (c) and (d)]. [(a)-(c)] Antisymmetric modes. [(b)-(d)] Symmetric modes. The contour levels $\widetilde{\omega}_{y} /\left|\widetilde{\omega}_{y \max }\right|$ shown are $\pm 0.1, \pm 0.3, \pm 0.5, \pm 0.7$, and \pm 0.9 for (a) and (b) and $\pm 0.05, \pm 0.1, \pm 0.3, \pm 0.5, \pm 0.7$, and \pm 0.9 for (c) and (d).

\section{Oscillatory instabilities}

At large Reynolds number $\operatorname{Re}_{\Gamma}=10^{5}$, the other unstable branches with smaller growth rate are visible in Fig. 2(a), between $k_{y} a=0.5$ and $k_{y} a=2.5$ with a maximum growth rate of 0.24 at $k_{y} a=1.09$ and between 2.8 and 5.6 with a maximum of 0.32 at 4.2 , they will be thereafter referred to as oscillatory instabilities since the growth rates have an imaginary part. They exist for both symmetric and antisymmetric modes with extremely close growth rates. Figure 5 shows the axial vorticity of the antisymmetric oscillatory modes for the most unstable wavenumber of each unstable bands, the symmetric oscillatory modes are not shown, since their eigenfunction is similar except that its vorticity perturbation field is antisymmetric. The vorticity perturbation at $k_{y} a=1.09$ [Fig. 5(a) for the real part and Fig. 5(b) for the imaginary part] consists of a central maximum located inside the core of each base flow vortex and two lobes of opposite signs at the periphery. The vorticity perturbation in each vortex core may schematically be constructed by superposition of a $m=0$ and $|m|=2$ azimuthal perturbation and may be interpreted as the elliptic instability mode resulting from a resonance between the strain and Kelvin waves of azimuthal wavenumbers $m=0$ and $|m|=2$ as proposed by Billant et al. ${ }^{8}$ in the case of the Lamb-Chaplygin dipole and investigated in details by Eloy and Le Dizès ${ }^{12}$ for the Rankine vortex.

The frequency of the most unstable oscillatory mode of the first branch has been compared to the frequency of the resonant Kelvin waves of azimuthal wavenumbers $m=0$ and $|m|=2$ on a Rankine vortex obtained by solving the disper-
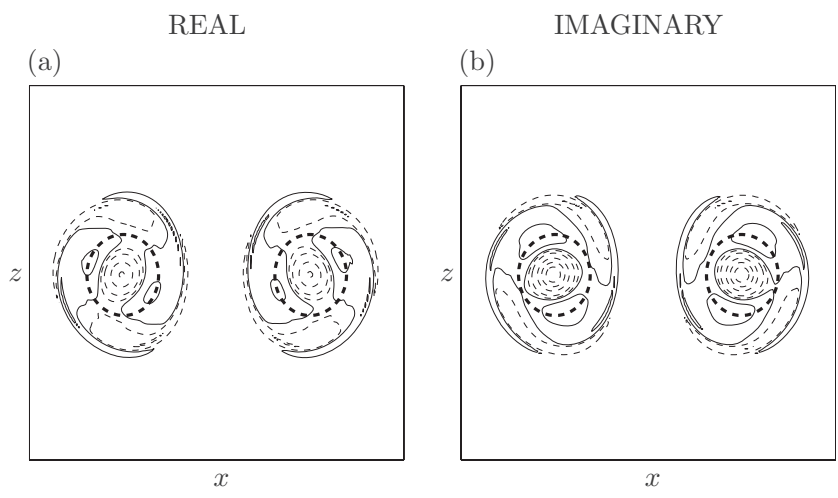

(c)

(d)
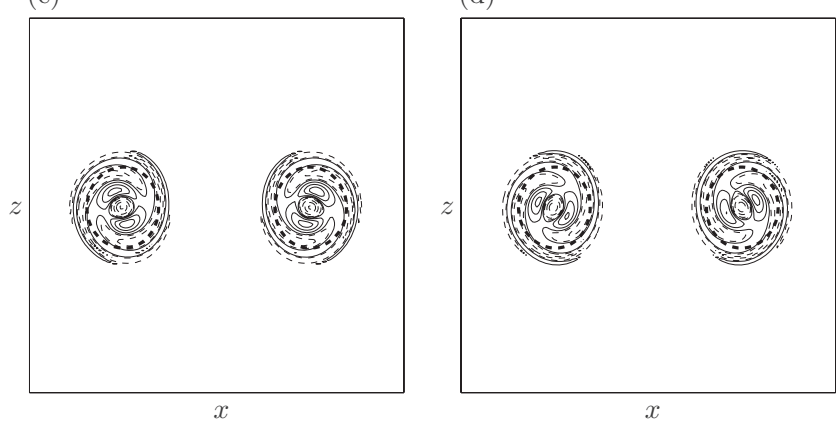

FIG. 5. Oscillatory instability: Same as Fig. 3 except that only the antisymmetric mode is presented. [(a) and (b)] $k_{y} a=1.09$ and [(c) and (d)] $k_{y} a=4.2$. Since the growth rate is complex, the eigenmode is complex. (a)(c) show the real part of the eigenmode axial vorticity. [(b)-(d)] Imaginary part of the modes. The contour levels $\widetilde{\omega}_{y} /\left|\widetilde{\omega}_{y \max }\right|$ shown are $\pm 0.02, \pm 0.1$, $0.2, \pm 0.3,-0.5$, and -0.7 .

sion relation given by $\operatorname{Saffman}^{32}$ (see also Billant et al. ${ }^{8}$ ). The first resonance occurs at the wavenumber $k_{y} a_{r}=1.24$ for a frequency $\sigma_{i} / \Omega=0.8$, where $a_{r}$ is the radius and $\Omega$ the rotation rate of the Rankine vortex. Since the sampling frequency $2 \pi / \Delta T$ used to retrieve the eigenmodes with the Krylov method explained in Sec. II C is very small compared to the frequency of the oscillatory modes, the imaginary parts of the eigenvalues of these modes computed with this method are not the very frequency but its harmonic with the sampling frequency. Therefore, the frequency of the propagative mode at the maximum of the first unstable band $k_{y} a=1.09$ has been computed directly from the temporal evolution of the energy $E$ of the perturbation sample at high frequency $2 \pi / 10^{-3}$. The energy oscillates at a period $T$ while it grows, giving a measured value $\sigma_{i} / \Omega(r=0)=\sigma_{i} 2 \pi a^{2} / \Gamma$ $=0.6$ at $k_{y} a=1.09$ for both symmetries, $\Omega(r=0)$ being the rotation rate at the center of the vortex. The agreement, with the predicted value $\sigma_{i} / \Omega=0.8$, is satisfactory, since the theory is valid at small ellipticity and considers Rankine vortices, whereas the deformation is large and the vorticity distribution Gaussian (Lamb-Oseen vortices) in the present case.

Eloy and Le Dizès ${ }^{12}$ showed that the inviscid growth rates of the resonant Kelvin modes combination $m=0$ and $m=2$ and the helical modes $m= \pm 1$ are comparable for the Rankine vortex. These oscillatory instabilities were not found by Sipp and Jacquin ${ }^{7}$ in their inviscid analysis. These authors put forward the presence of a viscous critical layer since, for the Lamb-Oseen vortex, the Kelvin waves for 
$m=2$ with frequencies between 0 and $2 \Omega_{\max }\left(\Omega_{\max }\right.$ the maximum of the angular velocity at the center of the vortex), presents a critical layer at the radius where the azimuthal phase velocity of the perturbation equals the angular velocity $\Omega(r)$ of the base state, i.e., $\omega / m=\Omega(r)$. This viscous critical layer induces a finite damping of the Kelvin waves when the Reynolds number goes to infinity. In the present stability analysis, this resonance is however observed for large Reynolds number suggesting that, for the present case, with finite ellipticity, the damping due to the critical layer induces a reduction of the growth rate, not sufficient to stabilize the mode. The possibility that finite ellipticity effects might dominate the viscous effects and change the nature of the critical layer is presently a conjecture that would deserve further analysis.

\section{NON-NORMALITY AND ADJOINT MODES}

The stability analysis presented above considers the eigenmode of the linearized evolution operator. For each axial wavenumber $k_{y}$, it is known that, starting from random initial condition, the flow will eventually converge toward the leading eigenmode and experience an exponential growth when this mode is unstable. This exponential longtime behavior should be complemented by examining the fate of the perturbations at short time since, as the Navier-Stokes operator linearized around the base flow is non-normal (Schmid and Henningson ${ }^{33}$ ), they may exhibit transient growth of their energy. A standard technique to compute transient growth requires the resolution of the adjoint of linearized Navier-Stokes equations. ${ }^{34}$ The inner product used to construct the adjoint is chosen as

$$
\begin{aligned}
\left\langle\mathbf{f}^{\prime} \mid \mathbf{f}\right\rangle & =\int_{0}^{\tau} \int_{0}^{L_{x}} \int_{0}^{L_{z}} \mathbf{f}^{\prime * T} \cdot \mathbf{f} d x d z d t \\
& =\int_{0}^{\tau} \int_{0}^{L_{x}} \int_{0}^{L_{z}}\left(\mathbf{u}^{\prime * T} \cdot \mathbf{u}+p^{\prime *} p\right) d x d z d t,
\end{aligned}
$$

where $\mathbf{f}^{\prime}=\left(\mathbf{u}^{\prime}, p^{\prime}\right)$ and $\mathbf{f}=(\mathbf{u}, p)$ are two complex state vectors, the superscripts ${ }^{*}$ and ${ }^{T}$ denote the complex conjugate and the transposition, and $L_{x}, L_{z}$ defined on Sec. II C. The kinetic energy is then given by

$$
E(t)=(\mathbf{u} \mid \mathbf{u})=\int_{0}^{L_{x}} \int_{0}^{L_{z}}\left(\mathbf{u}^{* T} \cdot \mathbf{u}\right) d x d z .
$$

\section{A. Adjoint equations and adjoint eigenmodes}

The adjoint of the linearized Navier-Stokes equations, is deduced from Eq. (5) using the Lagrange identity (Ince, ${ }^{35}$ Hill $^{36}$ ) and rewritten as

$$
\begin{aligned}
& \frac{\partial \mathbf{u}^{+}}{\partial t^{\prime}}=\boldsymbol{\omega}_{B} \times \mathbf{u}^{+}-\nabla \times\left(\mathbf{u}_{B} \times \mathbf{u}^{+}\right)-\nabla p^{+}+\nu \Delta \mathbf{u}^{+}, \\
& \nabla \cdot \mathbf{u}^{+}=0,
\end{aligned}
$$

where $\left[\mathbf{u}^{+}, p^{+}\right](x, y, z, t)$ are the adjoint velocity and pressure perturbations and $t^{\prime}=-t$. These equations are integrated with a pseudospectral technique similar to the technique used to
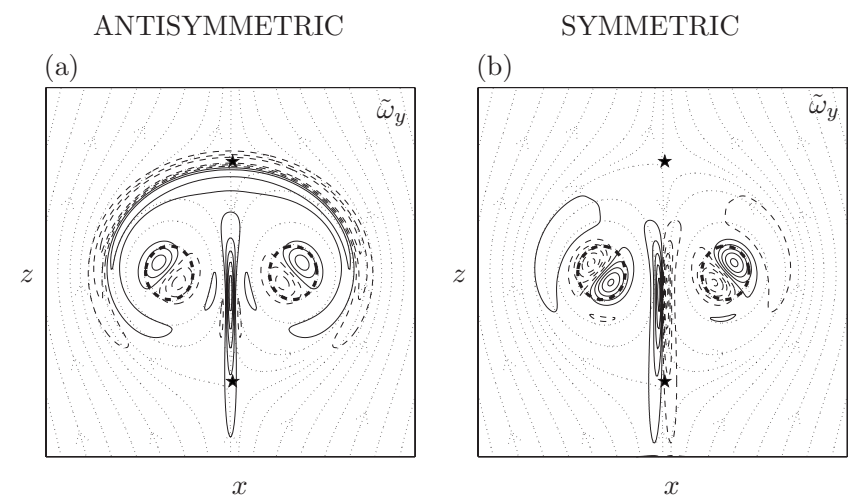

(c)

(d)
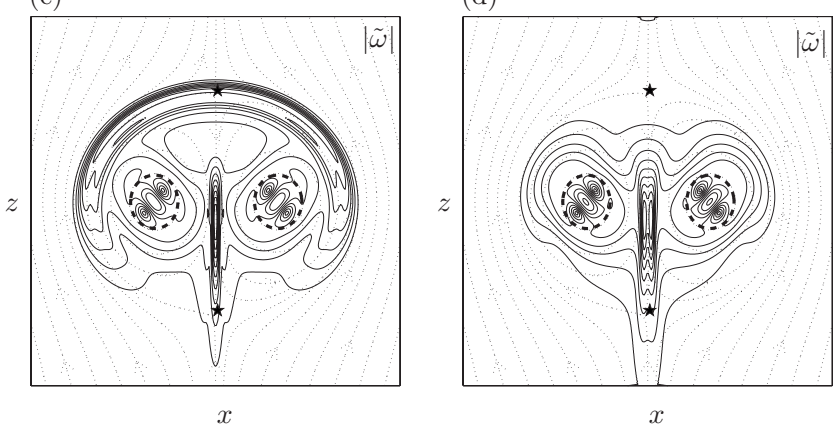

FIG. 6. Adjoint modes for the elliptic instability: Contours of axial vorticity $\widetilde{\omega}_{y}[(\mathrm{a})$ and (b)] and square root of enstrophy $|\widetilde{\omega}|[(\mathrm{c})$ and (d)] of adjoint eigenmodes in the $(x, z)$ plane for $\operatorname{Re}_{\Gamma}=2000$ at the elliptic instability maximum $k_{y} a=2.26$. [(a)-(c)] Antisymmetric mode. [(b)-(d)] Symmetric mode. Continuous lines correspond to positive vorticity and dashed lines correspond to negative vorticity. The contour levels shown in (a) and (b) are $\widetilde{\omega}_{y} /\left|\widetilde{\omega}_{y \max }\right|= \pm 0.1, \pm 0.3, \pm 0.7, \pm 0.9$ and $|\widetilde{\omega}| /\left|\widetilde{\omega}_{\max }\right|$ $=0.1,0.2,0.3,0.4,0.5,0.6,0.7,0.8,0.9$ in (c) and (d). The heavy dashed lines mark the vortex core of the base state like in Fig. 3. The stars represent the stagnation points of the base state and the dotted lines with arrows correspond to the streamlines of the base state. The size of the domain shown is larger than in previous figures: $3 b \times 3 b$.

solve the direct problem in Sec. II C, where the advection term is replaced by $\boldsymbol{\omega}_{B} \times \mathbf{u}^{+}-\nabla \times\left(\mathbf{u}_{B} \times \mathbf{u}^{+}\right)$. The size of the box and the time step are the same as for the direct linear Navier-Stokes equations. The leading eigenmodes of the adjoint operator are computed using the same Krylov method, the symmetries being imposed either at each time step or a posteriori as for the direct equations. For all $k_{y}$, the computed spectrum of the adjoint operator is equal to the direct spectrum with a fifth digit precision. It verifies also the biorthogonality property with the same accuracy: all adjoint and direct eigenmodes corresponding to different eigenvalues are orthogonal for the inner product defined in Eq. (12) (see Grosch and Salwen ${ }^{37}$ for a demonstration in the two dimensional).

Figure 6 displays the axial vorticity and the enstrophy of the antisymmetric and symmetric adjoint eigenmodes at the peak of the first elliptic instability branch for $\operatorname{Re}_{\Gamma}=2000$. The spatial distribution of the adjoint eigenmodes differs for both symmetries. For the antisymmetric mode, the vorticity perturbation of the adjoint elliptic mode is intense inside the core of the vortices and on the contracting manifold of both the upper and lower hyperbolic stagnation points. The symmetric mode is intense inside the core and on the contracting 
(a)

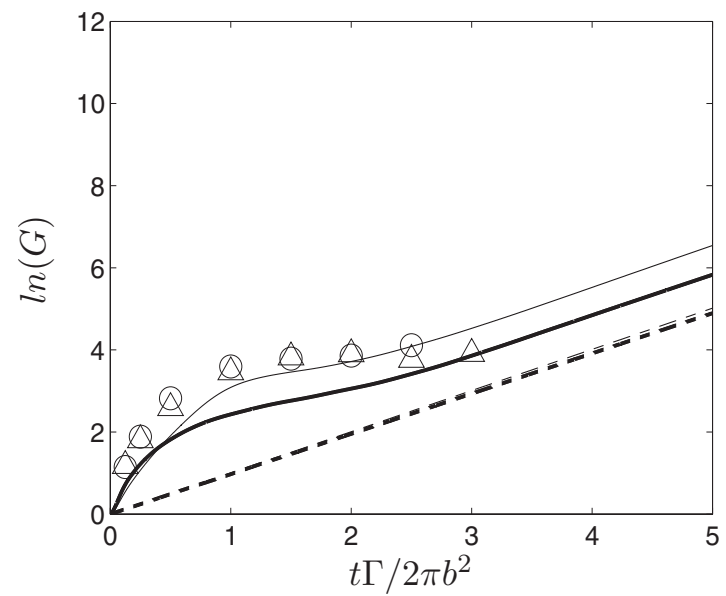

(b)

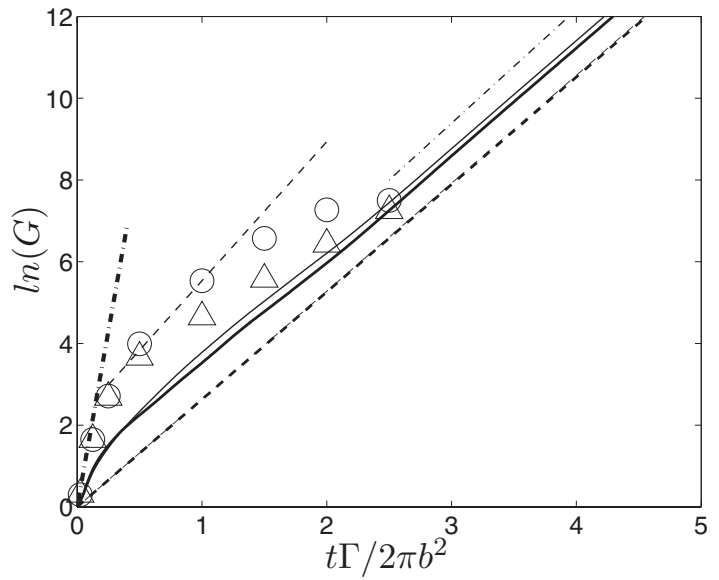

FIG. 7. Energy gain of the symmetric (heavy lines and $\triangle$ ) and antisymmetric (thin lines and $\bigcirc$ ) modes as function of the time $t$ nondimensionalized by the advection time of the dipole $2 \pi b^{2} / \Gamma$ for different initial conditions. (a) $\operatorname{Re}_{\Gamma}=2000$; (b) $\operatorname{Re}_{\Gamma}=10^{5}$. Dashed lines: initial condition is the direct eigenmode. Continuous lines: initial condition is the adjoint eigenmode, optimal at large times. Open symbols: optimal gain at each instant. In (b), heavy dashed-dotted lines: theoretical prediction of the energy gain at short times; dotted line: hyperbolic instability prediction of Caulfield and Kerswell (Ref. 38). Dashed-dotted line: elliptic instability prediction of Waleffe (Ref. 6).

manifold of the lower hyperbolic stagnation point only, with no contribution of the upper stagnation point.

\section{B. Large time behavior}

We investigate now the initial condition which maximizes the energy gain at large time $t$ defined by

$$
G(t)=\frac{E(t)}{E(0)}=\frac{(\mathbf{u}(t) \mid \mathbf{u}(t))}{(\mathbf{u}(0) \mid \mathbf{u}(0))}
$$

where $E(0)$ is the initial kinetic energy of the perturbation and $E(t)$ is its value at time $t$. Figures 7(a) and 7(b) show the logarithm of the energy gain as function of time for a single axial wavenumber at the peak of the elliptic instability $k_{y} a=2.26$, for different initial conditions and for two different Reynolds numbers $\operatorname{Re}_{\Gamma}=2000$ and $\operatorname{Re}_{\Gamma}=10^{5}$.

The heavy (thin) dashed line corresponds to the amplification factor in the symmetric (antisymmetric) case with the direct eigenmode taken as initial condition. The dependence of $\ln (G)$ for $k_{y} a=2.26$ as function of $t$ is linear and the slope is twice the growth rate computed previously, i.e., $\sigma_{s} 2 \pi b^{2} / \Gamma=0.49$ for the symmetric case and $\sigma_{a} 2 \pi b^{2} / \Gamma$ $=0.5$ for the antisymmetric case, for $\operatorname{Re}_{\Gamma}=2000$. Since the difference between the growth rate of the symmetric and antisymmetric modes is small, their energy differs only by $12 \%$ for $\operatorname{Re}_{\Gamma}=2000$ and less than $6.5 \%$ for $\operatorname{Re}_{\Gamma}=10^{5}$ at time $t \Gamma / 2 \pi b^{2}=5$. For $\operatorname{Re}_{\Gamma}=10^{5}$ and large time, the growth rate of the energy which is the slope of $\ln (G) \sigma 2 \pi b^{2} / \Gamma=1.32$, is in good agreement with the prediction of Waleffe ${ }^{6}$ [dasheddotted line on Fig. 7(b)]. Effectively, theoretical prediction of energy growth for an elliptic flow, in the framework of local inviscid theory, is $9 / 8 \epsilon=1.39$, with nondimensional $\epsilon$ evaluated in the core of the primary vortices [see Fig. 1(b)]. At large time and for $k_{y} a=2.26$ dynamics [Figs. 4(a) and 4(b)], and growth rate [Fig. 7(b)] are triggered by elliptic instability.

The heavy (thin) continuous line corresponds to the gain in the symmetric (antisymmetric) case when the initial condition is the adjoint eigenmode. The final energy gain at time $t \Gamma / 2 \pi b^{2}=5$, when initialized by the adjoint instead of the direct eigenmode, is larger by a factor of 4 (antisymmetric) and 2 (symmetric) for $\mathrm{Re}_{\Gamma}=2000$ and by a factor of 2.5 (antisymmetric) and a factor of 2 (symmetric) for $\operatorname{Re}_{\Gamma}=10^{5}$. The antisymmetric mode is therefore more sensitive to initial perturbation than the symmetric one, particularly at small Reynolds number. The adjoint eigenmode is the initial condition that maximizes the energy gain at large times $\left(\mathrm{Hill}^{36}\right)$. Effectively, any initial condition $\mathbf{u}$ with $\left(\mathbf{u}_{1}^{+} \mid \mathbf{u}\right) \neq 0$, where $\mathbf{u}_{1}^{+}$ is the leading adjoint mode, is dominated at large time by the leading eigenmode $\mathbf{u}_{1}$ with an amplitude equal to $\left(\mathbf{u}_{1}^{+} \mid \mathbf{u}\right) /\left(\mathbf{u}_{1}^{+} \mid \mathbf{u}_{1}\right) e^{\lambda_{1} t}$ which is the largest when $\mathbf{u}=\mathbf{u}_{1}^{+}$. Therefore, when both $\mathbf{u}_{1}^{+}$and $\mathbf{u}_{1}$ are normalized, $1 /\left|\left(\mathbf{u}_{1}^{+} \mid \mathbf{u}_{1}\right)\right|^{2}$ measures the extra gain obtained, at large time, by initializing by the adjoint mode $\mathbf{u}_{1}^{+}$instead of by the direct mode $\mathbf{u}_{1}$. Presently, for $\operatorname{Re}_{\Gamma}=2000$ (for $\operatorname{Re}_{\Gamma}=10^{5}$ ), its value is $\ln \left(1 /\left|\left(\mathbf{u}_{1}^{+} \mid \mathbf{u}_{1}\right)\right|^{2}\right)=1.6(0.85)$ in the antisymmetric case and $\ln \left(1 /\left|\left(\mathbf{u}_{1}^{+} \mid \mathbf{u}_{1}\right)\right|^{2}\right)=1.1(0.71)$ in the symmetric case, in close agreement with the numerical results of Fig. 7(a) [for Fig. 7(b)] obtained by direct time integration of the perturbation equation. The scalar $1 /\left(\mathbf{u}_{1}^{+} \mid \mathbf{u}_{1}\right)$ indicates the sensitivity to initial perturbation of the leading eigenmode and quantifies therefore its non-normality. However, at finite times, several eigenmodes may interfere to give large transient and the knowledge of the leading adjoint mode is not sufficient to characterize the finite time behavior of perturbations. 


\section{Short time behavior}

Transient growths are first computed for a single axial wavenumber $k_{y} a=2.26$ corresponding to the maximum of the first elliptic instability branch. We look for the optimal initial condition at finite time $\tau$, for both symmetries which maximizes the energy gain equation (14). Following Corbett and Bottaro, ${ }^{34}$ we define a propagator $\Phi(\tau)$,

$$
\mathbf{u}(\tau)=\Phi(\tau) \mathbf{u}(0),
$$

where $\Phi(\tau)$ expresses the evolution of an initial condition until $t=\tau, \mathbf{u}(\tau)$, obtained by integration of Eq. (5) with the initial condition $\mathbf{u}(0)$, hence the explicit form of the propagator is not required. The optimal gain at $t=\tau$ becomes from definition (14),

$$
\begin{aligned}
G(\tau) & =\max _{u(0)}\left(\frac{(\mathbf{u}(\tau) \mid \mathbf{u}(\tau))}{(\mathbf{u}(0) \mid \mathbf{u}(0))}\right) \\
& =\max _{u(0)}\left(\frac{\left(\Phi^{+}(\tau) \Phi(\tau) \mathbf{u}(0) \mid \mathbf{u}(0)\right)}{(\mathbf{u}(0) \mid \mathbf{u}(0))}\right),
\end{aligned}
$$

with $\Phi^{+}(\tau)$, the adjoint of $\Phi(\tau)$ for the inner product (12). It can be shown that $\Phi^{+}(\tau)$ is the propagator associated to the backward integration in time of Eq. (13) with the initial condition $\mathbf{u}^{+}\left(t^{\prime}=0\right)=\mathbf{u}(t=\tau)$ (see Andersson et al. ${ }^{39}$ in the spatial case). Therefore looking for the optimal initial condition and the optimal gain $G(\tau)$ (16) consists in finding the largest eigenvalue of the self-adjoint and positive operator $\Phi^{+}(\tau) \Phi(\tau)$, called the direct-adjoint propagator $\mathcal{P}$. The optimal initial condition is the corresponding eigenvector, and the optimal response the solution of Eq. (15) with $\mathbf{u}(0)$ the eigenvector.

The optimal perturbations can be computed by different techniques. We use here the direct-adjoint iterative procedure introduced by Luchini ${ }^{20}$ to determine the optimal initial condition and the optimal response at finite time for both symmetries. The direct equation (5) are integrated with the adjoint velocity perturbation taken as a guess value for the optimal initial condition: $\mathbf{u}(t=0)=\mathbf{u}_{1}^{+}$until time $t=\tau$. The backward in time integration of the adjoint equation (13) is then performed by taking the direct velocity perturbation at $t=\tau$ as initial condition: $\mathbf{u}^{+}\left(t^{\prime}=0\right)=\mathbf{u}(t=\tau)$, where $t^{\prime}=\tau-t$. The adjoint equation (5) is then integrated until $t^{\prime}=\tau$. Then, the procedure is reiterated taking as initial condition for the direct integration, the adjoint field at the final time $\tau$, normalized to unit energy: $\mathbf{u}(t=0)=\mathbf{u}^{+}\left(t^{\prime}=\tau\right) /\left|\mathbf{u}^{+}(t=\tau)\right|$. The successive integrations of direct and adjoint equations are repeated until the convergence is obtained, i.e., the variation of $\ln (G(\tau))$ is less than $10^{-2}$. This is usually achieved in about three to ten iterations. The choice of the adjoint eigenmode as an initial condition instead of a random noise, simply speeds up the convergence but does not change the final result. The optimal energy gains of symmetric $(\triangle)$ and antisymmetric $(\bigcirc)$ optimal perturbations obtained independently for each symmetry at time $\tau$ are displayed on Fig. 7 for $\operatorname{Re}_{\Gamma}=2000$ and $\operatorname{Re}_{\Gamma}=10^{5}$. They are very close for both symmetries. The optimal gains depart from the gain obtained with the adjoint mode as an initial condition, only until $t \Gamma / 2 \pi b^{2} \simeq 2.5$, that may be therefore considered as the dura-

$$
k_{y} a=2.26, \text { OPTIMIZATION TIME } t \Gamma / 2 \pi b^{2}=1
$$

Optimal perturbation

(a)

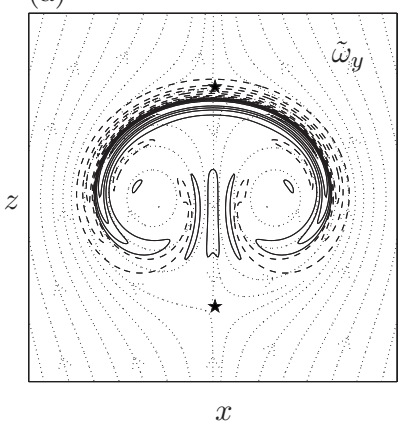

(c)

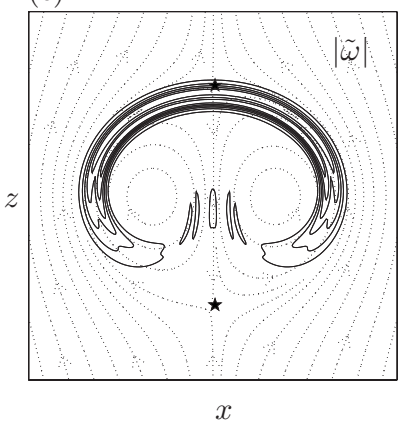

Optimal response

(b)

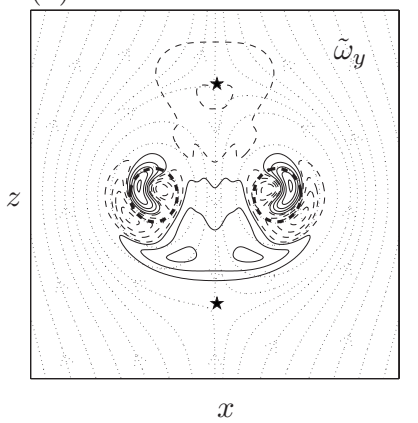

(d)

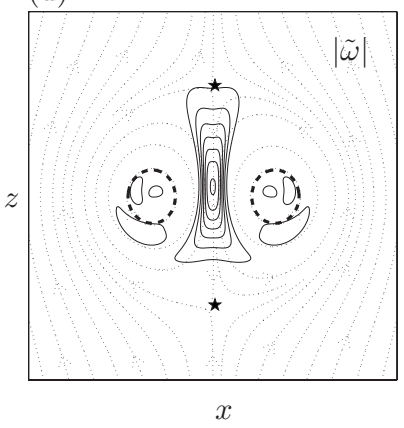

FIG. 8. Antisymmetric case, optimal initial perturbation [(a) and (b)] and optimal response [(c) and (d)] at $t \Gamma / 2 \pi b^{2}=1$ for the antisymmetric case. Contours of $[(\mathrm{a})-(\mathrm{c})]$ axial vorticity $\widetilde{\omega}_{y}$ and $[(\mathrm{b})-(\mathrm{d})]$ square root of enstrophy $|\widetilde{\omega}|$ in the $(x, z)$ plane for $\operatorname{Re}_{\Gamma}=2000$ at $k_{y} a=2.26$ for the antisymmetric case at $t \Gamma / 2 \pi b^{2}=1$. In (a)-(c), the continuous lines correspond to positive vorticity and the dashed lines correspond to negative vorticity. The contour levels shown are $\widetilde{\omega}_{y} /\|\widetilde{\mathbf{u}}(t=0)\|= \pm 0.01, \pm 0.05, \pm 0.09, \pm 0.13$, and 0.17 in (a), $\widetilde{\omega}_{y} /\|\tilde{\mathbf{u}}(t=0)\|= \pm 0.01, \pm 0.05, \pm 0.09, \pm 0.13$, and \pm 0.17 in (c), $|\widetilde{\omega}| /\|\widetilde{\mathbf{u}}(t=0)\|=0.02,0.05,0.08,0.11,0.14,0.17$, and 0.2 in (b) and $|\widetilde{\omega}| /\|\widetilde{\mathbf{u}}(t=0)\|=0.2,0.4,0.6,0.8,1.0,1.2$, and 1.4 in (d). Same as Fig. 6, for other characteristics.

tion of the transient regime. Two different leading mechanisms, explaining this transient behavior, may be identified, depending on the time for which the perturbation is optimized.

\section{Finite time dynamics}

The spatial distribution of the optimal initial perturbation and optimal response $t \Gamma / 2 \pi b^{2}=1$ are shown in Figs. 8 and 9 for $\operatorname{Re}_{\Gamma}=2000$ and are different for both symmetries. For the antisymmetric case, the axial vorticity of the initial optimal perturbation at $t \Gamma / 2 \pi b^{2}=1$ is intense on the contracting manifold of the upper stagnation point [Figs. 8(a) and 8(c)] whereas in the symmetric case it is strong between the two vortices, i.e., on the contracting manifold of the lower stagnation point [Figs. 9(a) and 9(c)]. The axial vorticity of the optimal response at $t \Gamma / 2 \pi b^{2}=1$ [Figs. 8(b) and 9(b)] is in both cases localized in the core of the vortices but, when comparing to Fig. 4, it has not yet converged toward the eigenmode with in particular, large enstrophy perturbation [Figs. 8(d) and 9(d)] outside the core of the vortices, confirming that the instant $t \Gamma / 2 \pi b^{2}=1$ considered here, is still in the transient regime. This optimal response is associated 


$$
k_{y} a=2.26, \text { OPTIMIZATION TIME } t \Gamma / 2 \pi b^{2}=1
$$

Optimal perturbation

(a)

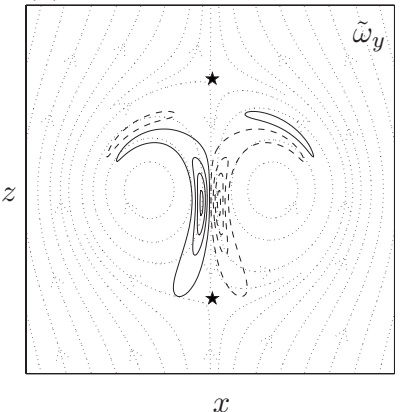

(c)

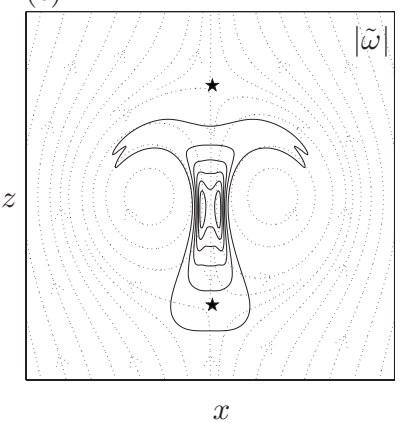

Optimal response (b)

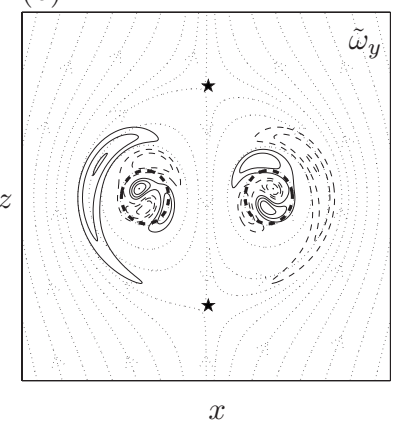

(d)

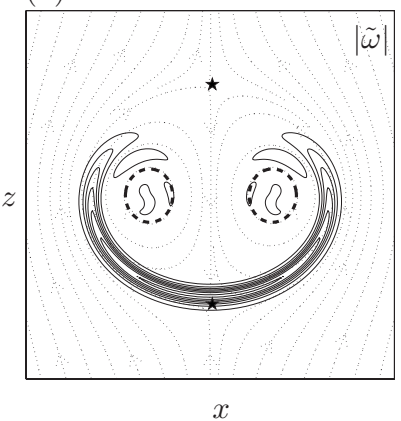

FIG. 9. Symmetric case, optimal initial perturbation and optimal response at $t \Gamma / 2 \pi b^{2}=1$. Same legend as Fig. 8. The contour levels shown are $\widetilde{\omega}_{y} /\|\widetilde{\mathbf{u}}(t=0)\|= \pm 0.01, \quad \pm 0.05, \quad \pm 0.09$, and \pm 0.13 in $(\mathrm{a}), \widetilde{\omega}_{y} /\|\widetilde{\mathbf{u}}(t=0)\|$ $= \pm 0.015, \pm 0.03, \pm 0.045$, and \pm 0.06 in $(\mathrm{c}),|\widetilde{\omega}| /\|\widetilde{\mathbf{u}}(t=0)\|=0.02,0.06,0.1$, $0.14,0.18,0.22$, and 0.26 in (b) and $|\widetilde{\omega}| /\|\widetilde{\mathbf{u}}(t=0)\|=0.1,0.25,0.4,0.55,0.7$, 0.85 , and 1 in (d).

with the formation of streamwise vortices (along $z$ ) on the symmetry axis in the antisymmetric case, i.e., along the stretching manifold of the upper hyperbolic point [Fig. 8(d)]. In the symmetric case, it corresponds to streamwise vortices nearly parallel to the $x$ axis, along the stretching manifold of the lower hyperbolic point [Fig. 9(d)]. For a pure inviscid extensional flow, Caulfield and Kerswell ${ }^{38}$ showed that the growth rate of the streamwise perturbation is equal to the strain $\epsilon_{h}$ evaluated at the hyperbolic point Fig. 1(b). This predicted slope has been plotted as a dotted line in Fig. 7(b) and compares well with the slope of $\ln \left(G(t)\right.$ for $\operatorname{Re}_{\Gamma}=10^{5}$ close to $t \Gamma / 2 \pi b^{2}=1$ [Fig. 7(b)], where Figs. 8 and 9 show that indeed one of the two hyperbolic points leads the optimal perturbation dynamics.

\section{Short time dynamics}

The perturbations, optimal at very early time, $t \Gamma / 2 \pi b^{2}$ $=0.025$, present identical features for symmetric and antisymmetric perturbations and only the later will be discussed (Fig. 10).

At very short times, the shape of the optimal initial perturbation is very similar to the optimal response since the flow has little time to evolve (Fig. 10). The enstrophy of the optimal perturbation and optimal response are concentrated very close to the points of maximal strain indicated by the black dots in Figs. 10(a) and 10(b) [the full strain field being

\section{$k_{y} a=2.26$, OPTIMIZATION TIME $t \Gamma / 2 \pi b^{2}=0.025$}

Optimal perturbation (a)

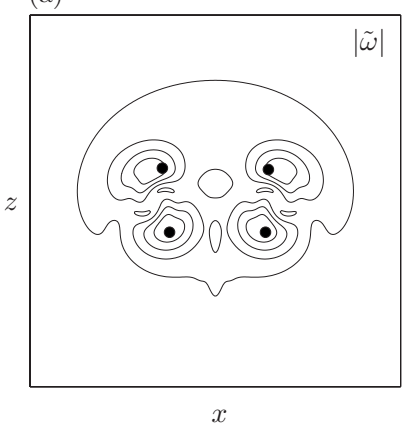

Optimal response

(b)

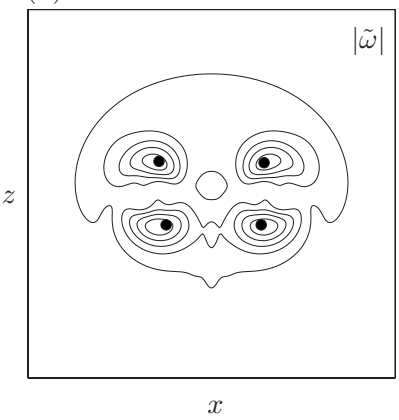

FIG. 10. Antisymmetric case: optimal initial perturbation (a) and optimal response (b) at $t \Gamma / 2 \pi b^{2}=0.025$. Contours of the square root of enstrophy $|\widetilde{\omega}|$ in the $(x, z)$ plane for $\operatorname{Re}_{\Gamma}=2000$ at $k_{y} a=2.26$. The contour levels shown in (a) and (b) are $|\widetilde{\omega}| /\|\widetilde{\mathbf{u}}(t=0)\|=0.01,0.04,0.07,0.09$, and 0.12. The black dots correspond to the points of maximum strain of the base state.

plotted on Fig. 1(b)]. This localization of the optimal short time perturbation can be understood by extending the work of Caulfield and Kerswell ${ }^{38}$ who have shown, on an inviscid infinite flow model with hyperbolic streamlines and uniform strain, that the maximal energy gain at short times depends only on the strain $\epsilon$ of the flow. If we neglect the pressure as in Caulfield and Kerswell, ${ }^{38}$ the direct equation (5) and adjoint equation (13) read

$$
\frac{\partial \mathbf{u}}{\partial t}=-L_{B}(\mathbf{u}), \quad \frac{\partial \mathbf{u}^{+}}{\partial t}=-L_{B}^{+}\left(\mathbf{u}^{+}\right),
$$

with $L_{B}(\mathbf{u})=\mathbf{u}_{B} \times \boldsymbol{\omega}+\mathbf{u} \times \boldsymbol{\omega}_{B}$ and $L_{B}^{+}\left(\mathbf{u}^{+}\right)=\boldsymbol{\omega}_{B} \times \mathbf{u}^{+}-\nabla \times\left(\mathbf{u}_{B}\right.$ $\times \mathbf{u}^{+}$). At short times, Eqs. (17) integrate into

$$
\begin{aligned}
& \mathbf{u}(t)=\left(1-t L_{B}\right) \mathbf{u}(0)+O\left(t^{2}\right), \\
& \mathbf{u}^{+}(t)=\left(1-t L_{B}^{+}\right) \mathbf{u}^{+}(0)+O\left(t^{2}\right)
\end{aligned}
$$

the direct $\Phi(t)$ and adjoint $\Phi^{+}(t)$ defined in Eq. (15) are then approximately known at short time. The energy gain writes

$$
\begin{aligned}
G(t) & =1-\frac{t\left(\left(L_{B}+L_{B}^{+}\right) \mathbf{u}(0) \mid \mathbf{u}(0)\right)}{(\mathbf{u}(0) \mid \mathbf{u}(0))}+O\left(t^{2}\right) \\
& =1-2 t \frac{\left(S_{B} \mathbf{u}(0) \mid \mathbf{u}(0)\right)}{(\mathbf{u}(0) \mid \mathbf{u}(0))}+O\left(t^{2}\right),
\end{aligned}
$$

since $\left(L_{B}+L_{B}^{+}\right) \mathbf{u}(t)=2 S_{B} \mathbf{u}(t)$, with $S_{B}$ the symmetric part of the base flow velocity gradient tensor. At each location $(x, z)$, $S_{B}$ admits two eigenvalues $\pm \epsilon(x, z)$ which correspond to the local strain of the base flow represented in Fig. 1(b). The initial condition which maximizes the energy gain at short times is thus given by $\mathbf{u}(0)$ localized at the point where $\epsilon(x, z)$ is maximum and the gain is then

$$
\ln (G(t))=-2 \max (\epsilon) t+O\left(t^{2}\right) .
$$

This theoretical prediction of the energy gain at short times is reported in Fig. 7(b) by a heavy dashed-dotted line. At $t \Gamma / 2 \pi b^{2}=0.025$, for $\operatorname{Re}_{\Gamma}=2000$, the theory predicts $\ln (G)$ $=0.43$ whereas the numerical calculations give $\ln (G)=0.23$ 
(a)

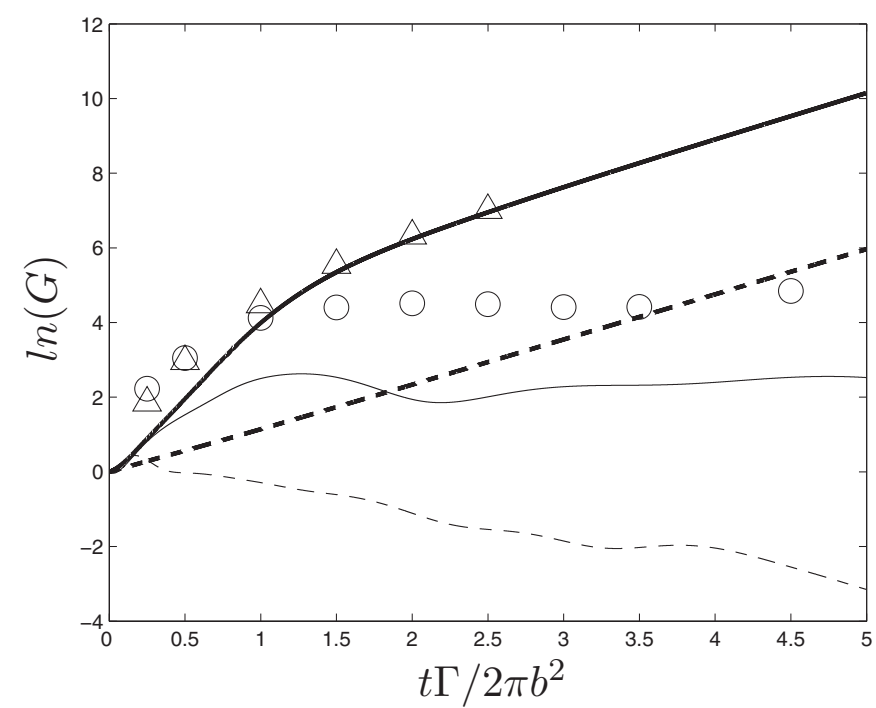

(c)

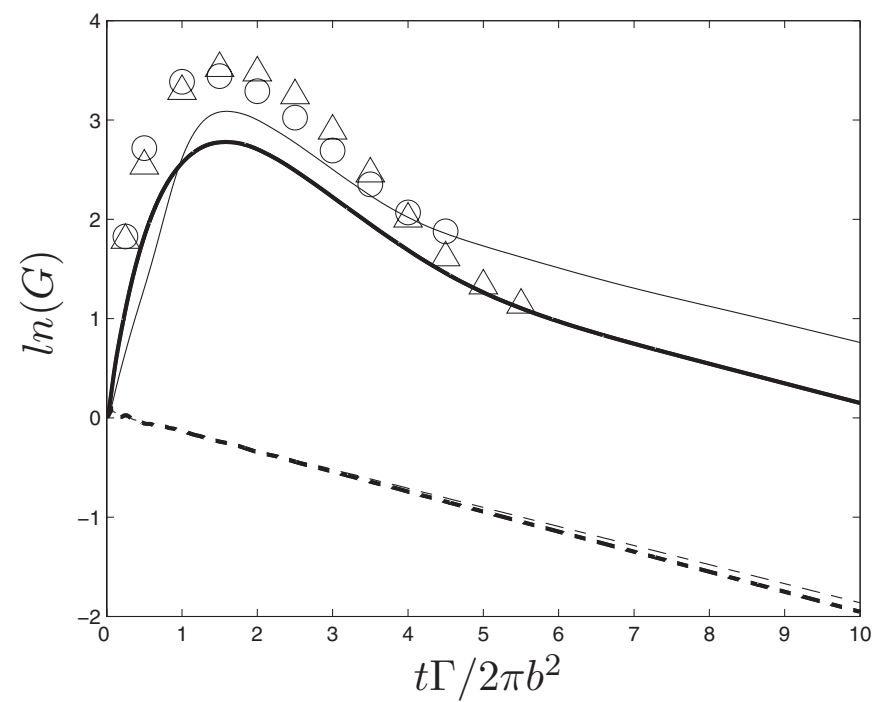

$k_{y} a=1.09$

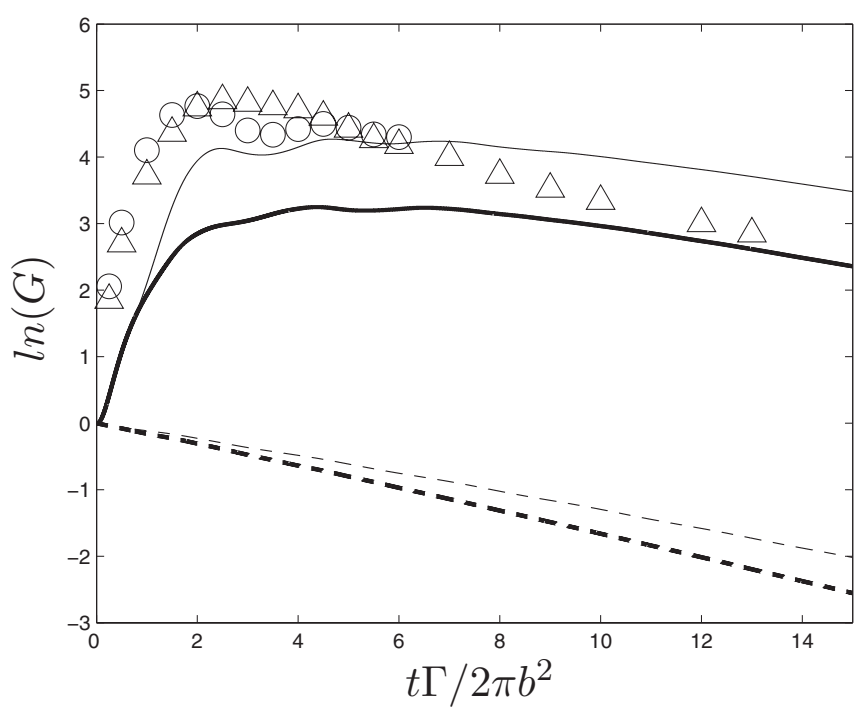

(d)

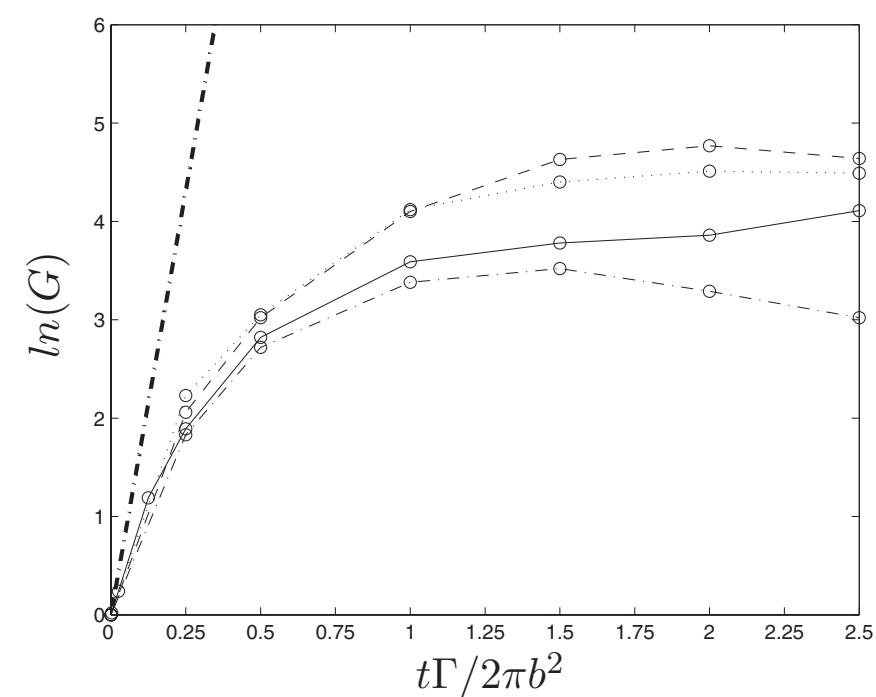

FIG. 11. Transient growth for different wavenumbers- $[(\mathrm{a})-(\mathrm{c})]$ same as Fig. $7(\mathrm{a})$. Energy gain at $\operatorname{Re}_{\Gamma}=2000$ of the symmetric (bold lines and $\triangle$ ) and antisymmetric (thin lines and $\bigcirc$ ) modes as function of the time $t$ nondimensionalized by $2 \pi b^{2} / \Gamma$ for different initial conditions. (a) $k_{y} a=0.2$, (b) $k_{y} a=1.09$, and (c) $k_{y} a=2.6$. (d) Close-up view of the optimal energy gain for the antisymmetric case (○) at short times for $k_{y} a=0.2(\cdots), k_{y} a=1.09(---)$, $k_{y} a=2.26(-)$, and $k_{y} a=2.6(-\cdot-)$. The bold dashed-dotted line corresponds to the theoretical prediction for the early instant optimal growth.

for the antisymmetric mode and $\ln (G)=0.26$ for the symmetric mode and, for $\operatorname{Re}_{\Gamma}=10^{5}, \ln (G)=0.30$ for the antisymmetric mode and $\ln (G)=0.32$ for the symmetric mode. At $t \Gamma / 2 \pi b^{2}=0.025$, the optimal perturbation and response (Fig. 10) are not totally concentrated on the points of maximal strain. The discrepancy certainly comes from the fact, that the theory is asymptotically valid for large $k_{y} a$ since, to neglect pressure at leading order, the perturbation should varies more rapidly along the $y$ direction than in the $(x, z)$ plane (approximation of the so-called pressureless dynamics ${ }^{40}$ ). For finite $k_{y}$, the pressure term in the expression of $L_{B}$ and $L_{B}^{+}$ cannot be neglected and it is already satisfactory that the asymptotic theory gives a decent agreement both in the localization of the perturbation and on the value of the gain.

\section{OPTIMAL ENERGY GAIN FOR DIFFERENT WAVENUMBERS}

Up until now, the optimal gain has been presented only for the most unstable wavenumber $k_{y} a=2.26$, but the procedure may be repeated for any $k_{y}$. The task is then formidable, since the optimal energy gain may be computed for each $k_{y} a$ at each time for both symmetries of the perturbation. We restrict ourselves to the intermediate Reynolds number $\operatorname{Re}_{\Gamma}=2000$ and close to, the maximum of the Crow instability $k_{y} a=0.2$, and on both sides of the first elliptic instability peak where the flow is stable for $\operatorname{Re}_{\Gamma}=2000$ for $k_{y} a=1.09$ and $k_{y} a=2.6$ [Fig. 2(b)]. Each of Figs. 11(a)-11(c) are similar to Fig. 7(a) but for these three new values of $k_{y} a$; Fig. 11(d) being a close up for early time of the optimal gain 
for the four wavenumbers plotted on Figs. 7(a) and 11(a)11(c). For all the stable wavenumbers, the maximum gain is reached around $t \Gamma / 2 \pi b^{2}=2$ with an energy increase by a factor close to 50. At very short time, Fig. 11(d) shows that all the wavenumbers computed experience a similar transient growth, no matter if they are stable or unstable at large time. This demonstrates that the sharp selection of unstable wavenumbers, predicted by the modal analysis [Fig. 2(b)] prevails only at very long time, but not at finite time when the shorter the optimization time considered, the smaller the difference in energy gain between different wavenumbers [Fig. 11(d)]. In particular, all the wavenumbers considered here are still experiencing an energy gain greater than one at time $t \Gamma / 2 \pi b^{2}=10$. In experiments, instabilities are observed at relatively short times. ${ }^{19}$ They may be still affected by transient effects, and therefore may not yet reflect the modal frequency selection valid at large time.

Brion et $a .^{23}$ investigated the energy gain of the Crow instability for the particular wavenumber $k_{y} a=0.2$ and $\operatorname{Re}_{\Gamma}=3600$, when the initial condition is the adjoint symmetric mode. They pointed out the importance of the lower hyperbolic point in the dynamics at large time, since the adjoint eigenmode is intense along the contracting manifold of that point. We retrieve these large time results, ${ }^{23}$ with an energy about 50 times larger when initialized by the adjoint mode than by the direct mode [Fig. 11(a)], and an instantaneous growth rate larger than its asymptotic value until $t \Gamma / 2 \pi b^{2}$ $=1.5$. Presently, we compute also the optimal gain of energy for the antisymmetric mode, not considered in. ${ }^{23}$ Remarkably, up to $t \Gamma / 2 \pi b^{2}=2.5$, the transient growth of antisymmetric perturbation, marked by an open circle in Fig. 11(a), is intense and nearly as large as the one of symmetric perturbation, even though they are stable at large time and decays, whereas the symmetric mode keeps increasing at large time when the Crow instability develops. At early instant up to $t \Gamma / 2 \pi b^{2}=1$, optimal energy gain for antisymmetric perturbations is even equal or larger than the symmetric one [Fig. 11(a)].

The enstrophy of the symmetric optimal perturbation, leading to the optimal energy gain at $t \Gamma / 2 \pi b^{2}=1$, represented on Fig. 12(c), is intense in the contracting manifold of the lower hyperbolic point as already pointed out by. ${ }^{23}$ However, axial vorticity, Fig. 12(a) exhibits spirals of vorticity surrounding the base vortices, suggesting the influence of a mechanism similar to that observed for a single vortex by Antkowiak and Brancher ${ }^{21}$ (comparison with Ref. 21 is postponed at the concluding section). The perturbation then evolves toward the usual Crow instability mode [Fig. 12(b)] whose axial vorticity is comparable to the one obtained at long time (Fig. 3). The antisymmetric dynamics is triggered by the upper hyperbolic point as observed in Figs. 13(a) and 13(c) which induces an antisymmetric displacement of the base flow vortices [Fig. 13(b)]. Optimal perturbations in the antisymmetric case place emphasis on the role of the upper hyperbolic point, not observed by Brion et al. ${ }^{23}$ who investigated the behavior of Crow instability only at large time and for the symmetric case.

$$
k_{y} a=0.2, \text { OPTIMIZATION TIME } t \Gamma / 2 \pi b^{2}=1
$$

Optimal perturbation

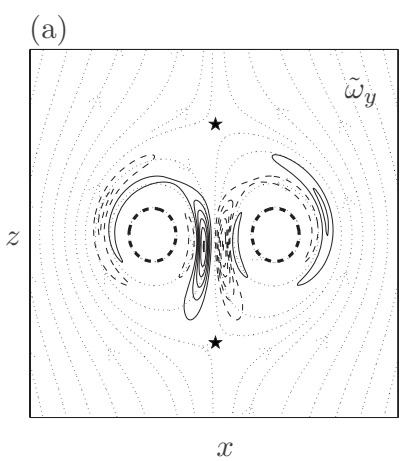

(c)

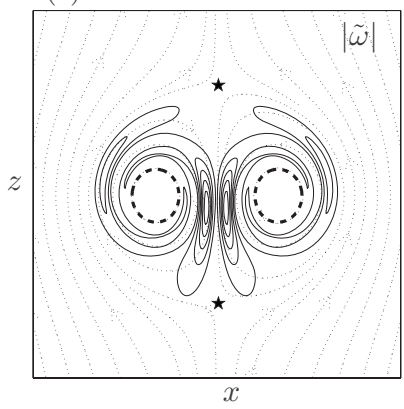

Optimal response

(b)

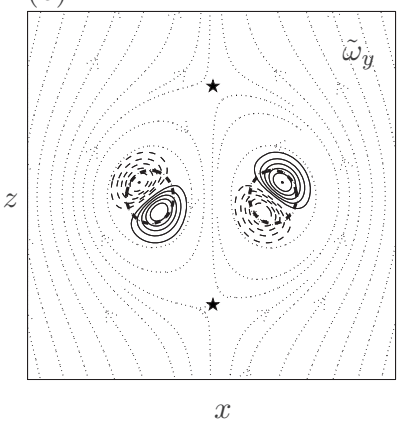

(d)

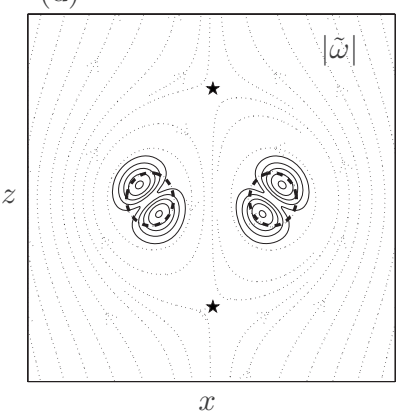

FIG. 12. Symmetric case, optimal initial perturbation [(a) and (b)] and optimal response [(c) and (d)] at $t \Gamma / 2 \pi b^{2}=1$ for $k_{y} a=0.2$ and $\operatorname{Re}_{\Gamma}=2000$. Axial vorticity $[(a)$ and (b)] and square root of enstrophy $|\widetilde{\omega}|[(\mathrm{c})$ and (d)]. The contour levels shown for (a) $\widetilde{\omega}_{y} /\|\widetilde{\mathbf{u}}(t=0)\|= \pm 0.01, \pm 0.02, \pm 0.03$, $\pm 0.04, \pm 0.05$, and \pm 0.06 ; in (b) are $\widetilde{\omega}_{y} /\|\widetilde{\mathbf{u}}(t=0)\|= \pm 0.02, \pm 0.08, \pm 1.2$, \pm 0.16 , and $\pm 12, \pm 0.9$ and $|\widetilde{\omega}| /\left|\widetilde{\omega}_{\max }\right|=0.02,0.06,0.1,0.14,0.18$ in (c) and $0.2,0.4,0.6$, and 0.81 in (d).

\section{DISCUSSION AND CONCLUSION}

For a spanwise homogeneous vortex dipole with relatively concentrated vorticity $(a / b=0.2)$, the long time and the transient growth given by the linear stability theory has been analyzed for all the spanwise wavenumbers. Long time dynamics is determined by the unstable modes that have been determined with a linear three-dimensional stability analysis based on a Krylov technique. The long-wavelength Crow instability has been retrieved. This instability is symmetric with respect to the plane separating the two vortices and the most unstable wavelength is $6.8 b$, which is in good agreement with Crow's theory. Both symmetric and antisymmetric modes of the elliptic instability with nearly identical growth rates have been found. These instability modes are nonoscillatory and very selective, with thin unstable band in wavenumber space well predicted by Tsai and Widnall, ${ }^{3}$ theory refined by Le Dizès and Laporte. ${ }^{10}$ The present results for $\operatorname{Re}_{\Gamma}=10^{5}$ are comparable with the inviscid linear stability analysis of Sipp and Jacquin ${ }^{7}$ but differs for the novel oscillatory modes, which has been obtained here for both symmetries, that were not observed in Ref. 7 but which is reminiscent to the one obtained by Billant et al. ${ }^{8}$ for the LambChaplygin dipole. Those unstable branches encompass broad ranges of wavenumbers, with a growth rate approximately one third of the leading elliptic modes which therefore dominate the leading instabilities of many 
$k_{y} a=0.2$, OPTIMIZATION TIME $t \Gamma / 2 \pi b^{2}=1$
Optimal perturbation

(a)

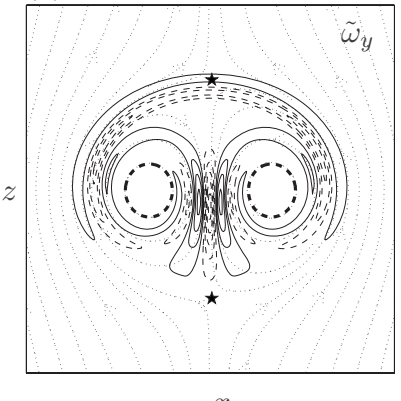

(c)

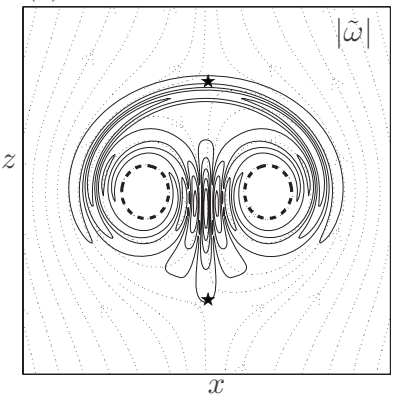

Optimal response (b)

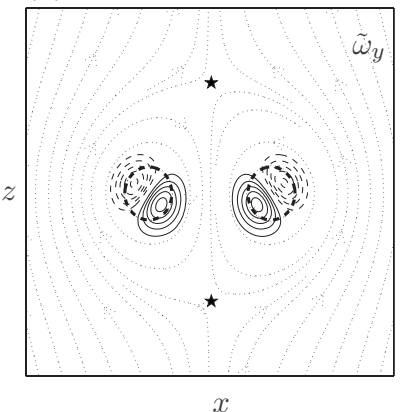

(d)

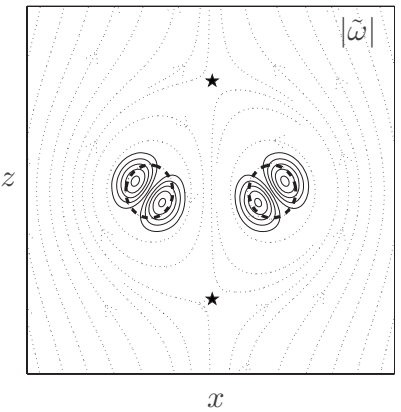

FIG. 13. Antisymmetric case, optimal initial perturbation [(a) and (b)] and optimal response [(c) and (d)] at $t \Gamma / 2 \pi b^{2}=1$ for $k_{y} a=0.2$ and $\operatorname{Re}_{\Gamma}=2000$. Axial vorticity in (a) and (b) and square root of enstrophy $|\widetilde{\omega}|[(\mathrm{c})$ and (d)]. The contour levels shown in (a) are $\widetilde{\omega}_{y} /\|\widetilde{\mathbf{u}}(t=0)\|= \pm 0.05, \pm 0.1, \pm 0.15$, $\pm 0.2, \pm 0.25$, and \pm 0.3 ; in (b) are $\widetilde{\omega}_{y} /\|\widetilde{\mathbf{u}}(t=0)\|= \pm 0.02, \pm 0.08, \pm 1.2$, \pm 0.16 , and \pm 12 and $|\widetilde{\omega}| /\left|\widetilde{\omega}_{\max }\right|=0.02,0.06,0.1,0.14,0.18$ in (c) and $0.2,0.35,0.5,0.65$, and 0.8 in (d).

wavevectors. This oscillatory instability seems to result from the resonance between the strain and Kelvin waves of azimuthal wavenumbers $m=0$ and $|m|=2$ even though the latter is, in the absence of strain, strongly damped due to the presence of critical layers (Fabre et $a l^{41}$ ). The present results suggest that for finite strain, the critical layer might be modified and not regularized by the viscosity but by the strain.

Transient growth has been computed solving an optimization loop by integrating alternatively the direct and adjoint linearized Navier-Stokes equation. ${ }^{34}$ For all the wavenumbers, the transient regime lasts until the slope of the optimal gain versus time reaches the asymptotic slope given by the stability theory and where the optimal response is close to the unstable mode; $t \Gamma / 2 \pi b^{2}=2.5$ for unstable and nonoscillatory modes and larger time for oscillatory and stable modes (about $t \Gamma / 2 \pi b^{2}=12$ for stable oscillatory modes). For shorter optimization instant and depending upon the time at which the gain is computed, different regions of the base flow are active. For very short time compared to the advection time of the dipole, optimal perturbations are localized around the points of maximum strain, which are situated inside the cores in nontrivial locations, off the center, and are where perturbations grows the fastest, experiencing the strongest stretching. For time comparable to the advection time, the optimal growth is due to the transient hyperbolic instability of the upper (trailing) hyperbolic point for the antisymmetric case and the lower (leading) hyperbolic point for the symmetric mode: the perturbations are intensified by a stretching when trajectories pass close to one of the hyperbolic points. Optimal perturbations and response are then respectively on the contracting and stretching manifolds of the hyperbolic point.

It is interesting to compare the present results for two Lamb-Oseen vortices with $a / b=0.2$ with the newly transient growth mechanism identified on a single vortex. ${ }^{21}$ Antkowiak and Brancher ${ }^{21}$ found an intense amplification of kinetic energy for a wavenumber around $k_{y} a=1.4$ and large Reynolds number. The optimal initial perturbation is a set of spirals at the periphery of the Lamb-Oseen vortex, corresponding to a $m=1$ disturbance. During the evolution, until the optimal nondimensional time $\tau$, corresponding here to $\tau \Gamma / 2 \pi b^{2}=2$, a mechanism similar to Orr mechanism leads an intensification for the velocity perturbation link to the unfolding of the spirals followed by a contamination of the core through velocity induction. In the present case of two vortices, at time $t \Gamma / 2 \pi b^{2}=2$ and for $k_{y} a=1.09$, which is one of the closest value to $k_{y} a=1.4$ computed here, and at large Reynolds number $\operatorname{Re}_{\Gamma}=10^{5}$, the asymptotic behavior corresponding here to the oscillatory mode, Figs. 5(a) and 5(b), is not yet reached and the dynamics is still in the transient regime. To compare the instability mechanism of a single vortex and a vortex dipole, we have computed not only the leading optimal perturbation but the second optimal perturbation (with a lower energy gain) proceeding as follows: for $k_{y} a=1.09$, during the iterative procedure (Sec. III C) used to compute the optimal gain (16) at time $t \Gamma / 2 \pi b^{2}=2$ the series of fields $\left\{\mathbf{u}(0), \mathcal{P} \mathbf{u}(0), \mathcal{P}^{2} \mathbf{u}(0), \ldots, \mathcal{P}^{n} \mathbf{u}(0)\right\}$, where $\mathcal{P}=\Phi^{+}(\tau) \Phi(\tau)$ the direct-adjoint propagator, (16) $\Phi(\tau)$ propagator (15), and $n$ the number of iteration, are used to built a Krylov space and an Arnoldi procedure is implemented in order to retrieve approximations of leading eigenvalues of $\mathcal{P}$. The axial vorticity of the most amplified eigenvector of $\mathcal{P}$, corresponding to the optimal initial perturbation is represented in Fig. 14(a). The contribution of the hyperbolic point is dominant with large perturbations amplitude on the contracting manifold of the hyperbolic point but weak spirals around the primary vortices are also observed. The associated optimal response Fig. 14(c), is a dipolar structure corresponding at leading order to a $m=1$ perturbation of the core of each vortex. Therefore if the optimal perturbation differs between a dipole and a single vortex, the optimal response is similar, showing that for that particular instant and at that particular wavenumber away from the $m=1$ elliptic resonance, the mechanism described by Refs. 21 and 22 is active but modified, since the spiral initial perturbation wrapped around the single vortex and spirals out to reach the contracting manifold of the hyperbolic point for the dipole. The eigenmode of the direct-adjoint propagator $\mathcal{P}$ with the second largest eigenvalue corresponding to the second leading transient mode is less amplified $[\ln (G)=0.68$ for the dominant transient mode and $\ln (G)=0.62$ for the second transient mode], and is plotted in Fig. 14(b). This subdominant mode resembles the dominant one except that going around one of the two vortices the initial and final perturba- 


$$
k_{y} a=1.09, \text { OPTIMIZATION TIME } t \Gamma / 2 \pi b^{2}=2
$$

(a)

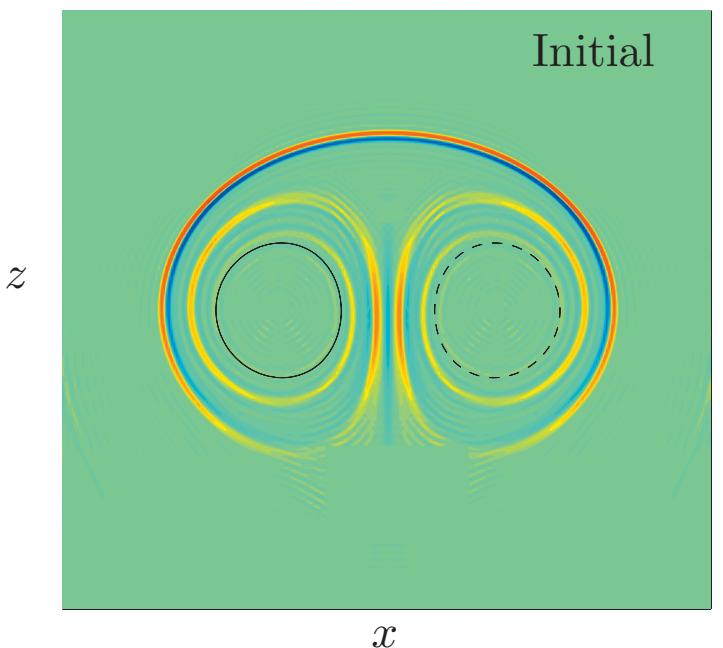

(c)

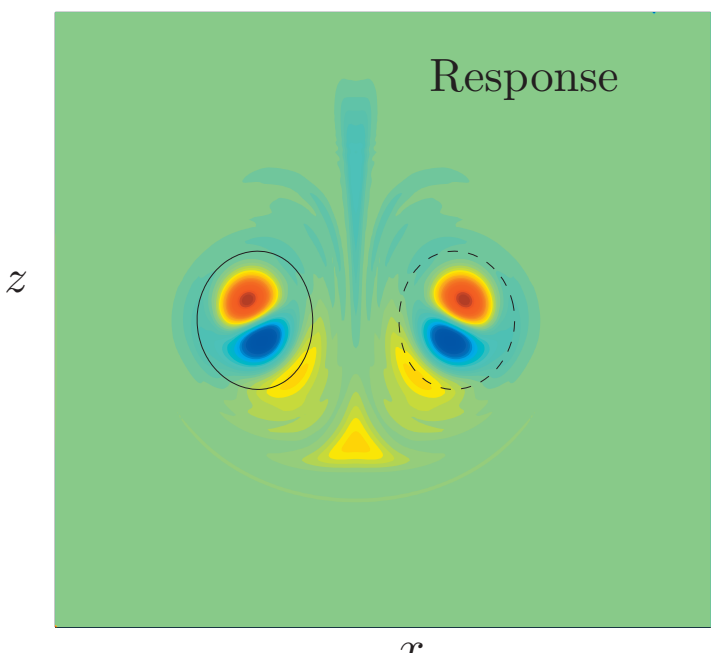

(b)
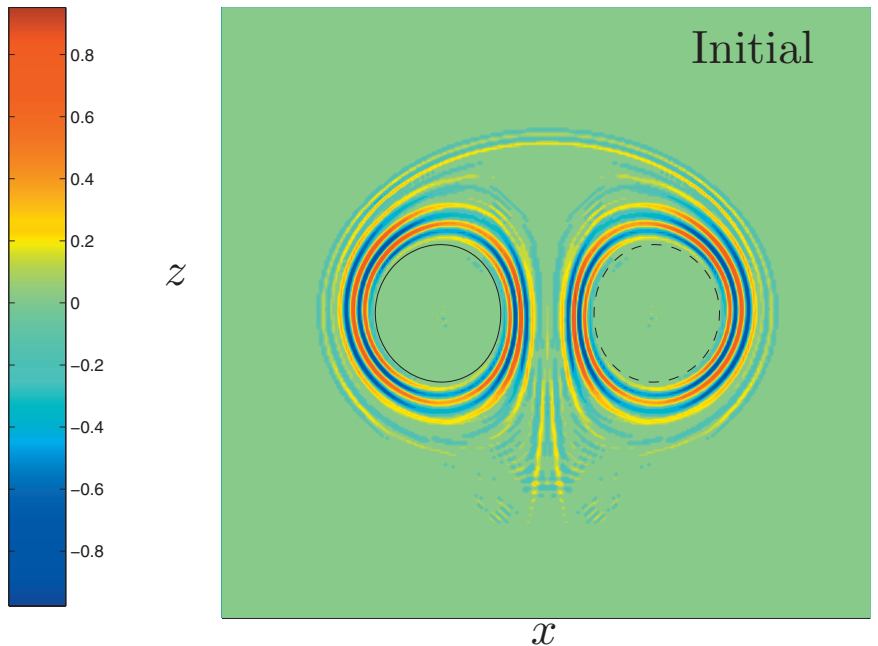

(d)
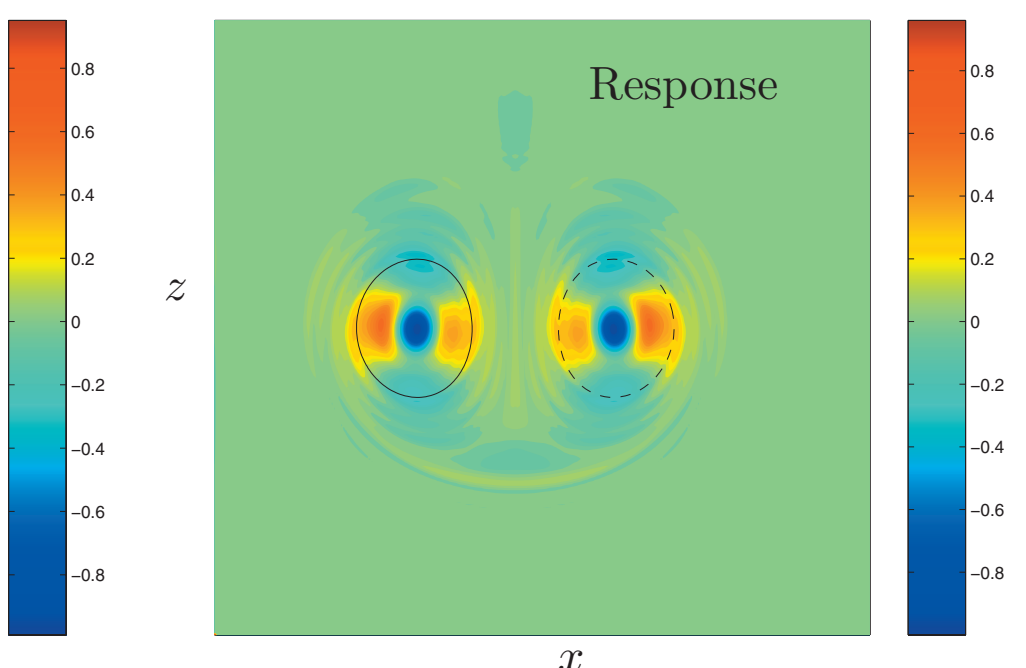

FIG. 14. (Color) Optimal initial perturbation [(a) and (b)] and optimal response [(c) and (d) $]$ at $t \Gamma / 2 \pi b^{2}=2$ in the antisymmetric case. Contours of axial vorticity $\widetilde{\omega}_{y}$ in the $(x, z)$ plane for $\operatorname{Re}_{\Gamma}=10^{5}$ at $k_{y} a=1.09$ for the antisymmetric case at $t \Gamma / 2 \pi b^{2}=2$. The continuous black lines correspond to primary vortices of positive vorticity and the dashed black lines correspond to negative vorticity of the primary vortices. Perturbation and response (a)-(c) for the leading optimal mode, and (b)-(d) for the second suboptimal mode.

tions oscillate twice instead of one. More precisely, the axial vorticity of the initial perturbation consists of a double spiral surrounding each base flow vortex. The response Fig. 14(d), is made of a $m=0$ perturbation inside the core of each vortex surrounded by four lobes of alternate sign of vorticity, which may be identified as a $m=2$ perturbation. The initial perturbation is similar to the optimal spiral found by Ref. 21, but here for $m=2$ and not $m=1$, and the optimal response is more complex than the one discussed by Pradeep and Hussain ${ }^{22}$ since both $m=0$ and $m=2$ are present, probably coupled by the ellipticity of the base flow (which is absent for a single vortex). The fact that the growth is lower for this disturbance is in good agreement with Ref. 22 who found for the Oseen vortex a decrease of growth of energy when increasing the azimuthal wavenumber.

\section{APPENDIX A: NUMERICAL METHOD FOR COMPUTING THE 2D BASE STATE AND DOMAIN REDUCTION FOR THE STABILITY PROBLEM}

The evolution of the velocity, the vorticity, and the pressure of the base state $\left[\mathbf{u}_{\mathbf{B}}, \boldsymbol{\omega}_{\boldsymbol{B}}, p_{B}\right](x, z, t)$ is governed by the 2D Navier-Stokes equations:

$$
\begin{aligned}
& \frac{\partial \mathbf{u}_{\mathbf{B}}}{\partial t}=\mathbf{u}_{\mathbf{B}} \times \boldsymbol{\omega}_{\mathbf{B}}-\nabla\left(p_{B}+\frac{\mathbf{u}_{\mathbf{B}}^{2}}{2}\right)+\nu \Delta \mathbf{u}_{\mathbf{B}}, \\
& \nabla \cdot \mathbf{u}_{\mathbf{B}}=0 .
\end{aligned}
$$

These equations are solved with a pseudospectral method in Cartesian coordinates with periodic boundary conditions. The velocity, the vorticity and the pressure are expressed in 
TABLE I. Computational accuracy of the growth rate $\sigma^{*}=\sigma_{r} 2 \pi b^{2} / \Gamma$ with respect to the size of the box $L=L_{x}=L_{z}$ and the resolution $\delta=L / N$, with $N=N_{x}=N_{z}$ for three axial wavenumbers: $k_{y} a=2.26$ (antisymmetric mode), $k_{y} a=1.09$ (antisymmetric mode), and $k_{y} a=0.2$ (symmetric mode). The Reynolds number is $\operatorname{Re}_{\Gamma}=10^{5}$. The bold values correspond to the reference values, which have been chosen for the computation of the modes in Sec. II D.

\begin{tabular}{lccccccccc}
\hline \hline & & $k_{y} a=2.26$ & \multicolumn{3}{c}{$k_{y} a=1.09$} & \multicolumn{3}{c}{$k_{y} a=0.2$} \\
\hline$L$ & $\mathbf{3 b}$ & $6 b$ & $3 b$ & $\mathbf{3 b}$ & $6 b$ & $3 b$ & $3 b$ & $\mathbf{6 b}$ & $12 b$ \\
$N$ & $\mathbf{2 5 6}$ & 512 & 512 & $\mathbf{2 5 6}$ & 512 & 512 & 256 & $\mathbf{5 1 2}$ & 1024 \\
$\delta$ & $\mathbf{0 . 0 2 3 4}$ & 0.0234 & 0.0117 & $\mathbf{0 . 0 2 3 4}$ & 0.0234 & 0.0117 & 0.0234 & $\mathbf{0 . 0 2 3 4}$ & 0.0234 \\
$\sigma^{*}$ & $\mathbf{1 . 3 1 8 9}$ & 1.3189 & 1.3196 & $\mathbf{0 . 2 4 2 9}$ & 0.2427 & 0.2430 & 0.6223 & $\mathbf{0 . 7 4 7 0}$ & 0.7447 \\
\hline \hline
\end{tabular}

Fourier space by application of the two-dimensional Fourier transform:

$$
\begin{aligned}
{\left[\mathbf{u}_{\mathbf{B}}, \boldsymbol{\omega}_{\boldsymbol{B}}, p_{B}\right](x, z, t)=} & \int\left[\hat{\mathbf{u}}_{\mathbf{B}}, \hat{\boldsymbol{\omega}}_{\boldsymbol{B}}, \hat{p}_{B}\right] \\
& \times\left(k_{x}, k_{z}, t\right) e^{i\left(k_{x} x+k_{z} z\right)} d k_{x} d k_{z},
\end{aligned}
$$

where $k_{x}$ and $k_{z}$ are the components of the wavevector $\mathbf{k}_{2 D}$. In spectral space, the Navier-Stokes equations (A1) become

$$
\frac{\partial \hat{\mathbf{u}}_{\mathbf{B}}}{\partial t}=\mathbf{P}\left(\mathbf{k}_{2 D}\right)\left[\widehat{\left[\mathbf{u}_{\mathbf{B}} \times \boldsymbol{\omega}_{\boldsymbol{B}}\right.}\right]-\nu \mathbf{k}_{2 D}^{2} \Delta \hat{\mathbf{u}}_{\mathbf{B}}
$$

where $\mathbf{P}\left(\mathbf{k}_{2 D}\right)$ is the projection operator on the space of divergence-free fields which, in Fourier space, may be expressed as a tensor with components $P_{i j}=\delta_{i j}-k_{i} k_{j} / \mathbf{k}_{2 \mathrm{D}}^{2}$. The term $\mathbf{u}_{\mathbf{B}} \times \boldsymbol{\omega}_{\boldsymbol{B}}$ is evaluated in the physical space. Time integration is carried out with a second-order Adams-Bashforth scheme whereas the dissipative term $\nu \Delta \mathbf{u}_{\mathbf{B}}$ is integrated exactly.

The size of the periodic box $L_{x}=L_{z}=12$ is large enough to minimize the effects of periodic boundary conditions ( $L_{x}=L_{z}=60 a_{0}$ ) and to adapt to the downward descent of the dipole. The mesh is Cartesian with $512 \times 512$ (and 1024 $\times 1024$ in some cases) collocation points equally spaced in the $x$ and $z$ directions with $\delta x=\delta z=0.023$. This number allows approximately 16 points in each vortex core. The time step is set to $\delta t=10^{-3}$, which is small enough to fulfill the Courant-Friedrich-Levy condition: $\delta x / \delta t=23>U_{\max }$, with the maximum velocity here equal to $U_{\max } \simeq \Gamma_{0} / 2 \pi a_{0}=5$. For the linear stability analysis performed in Sec. II, the eigenmodes have been computed in a smaller box by cropping the velocity and vorticity of the base state $\left[\mathbf{u}_{\mathbf{B}}, \boldsymbol{\omega}_{\boldsymbol{B}}\right](x, z)$. There is no periodicity problem since the perturbation alone is periodic in the small box.

\section{APPENDIX B: COMPUTATIONAL ACCURACY}

The accuracy and the convergence of the results have been tested in a larger box size: $L_{x}=L_{z}=6 b$ with $512 \times 512$ grid points and with a finer resolution: $L_{x}=L_{z}=3 b$ with 512 $\times 512$ for three typical axial wavenumbers: the first maximum of the elliptic instability $k_{y} a=2.26$, the first maximum of the oscillatory instability $k_{y} a=1.09$ and the maximum of the Crow instability $k_{y} a=0.2$ for $\operatorname{Re}_{\Gamma}=10^{5}$. The results of the tests are displayed in Table I. We observe that doubling the size of the computational domain without changing the resolution does not change the growth rate and doubling the resolution changes the value of the growth rate by less than $1 \%$ for the wavenumbers $k_{y} a=2.26$ and $k_{y} a=1.09$. For the smaller wavenumber $k_{y} a=0.2$, widening the periodic box to $L=6 b$ and keeping the same resolution changes the value of the growth rate by $12 \%$.

\section{APPENDIX C: VISCOUS DAMPING ESTIMATE OF THE ELLIPTIC INSTABILITY}

In Sec. II D 2, we have observed that the viscous theory of Le Dizès and Laporte ${ }^{10}$ predicts that all the modes of the elliptic instability are stable for $\operatorname{Re}_{\Gamma}=2000$. However, the present direct linear stability analysis gives a band of unstable modes corresponding to the elliptic instability with a maximum growth rate $\sigma_{r} 2 \pi b^{2} / \Gamma=0.5$ at the wavenumber $k_{y} a=2.26$. This discrepancy suggests that the viscous damping proposed by Le Dizès and Laporte ${ }^{10}$ overestimate the actual one by nearly a factor of 2 when figures are analyzed. In this section, we give an estimate of the viscous damping using the adjoint eigenmode at the maximum of the elliptic instability at large Reynolds number. The viscosity is considered as a perturbation of the large Reynolds number linear Navier-Stokes operator (Schmid and Henningson ${ }^{33}$ ): $L \sim L^{(0)}+\nu L^{(1)}$, where $L$ is the viscous linear Navier-Stokes operator, $L^{(0)}$ the inviscid linear Navier-Stokes operator, $L^{(1)}=\Delta_{2 D}-k_{y}^{2}$ the perturbation operator. Asymptotic expansion of the leading eigenvalue $\sigma_{1} \sim \sigma_{1}^{(0)}+\nu \sigma_{1}^{(1)}$ and eigenmode $\phi_{1} \sim \phi_{1}^{(0)}+\nu \phi_{1}^{(1)}$, where $\sigma_{1}^{(0)}\left(\phi_{1}^{(0)}\right)$ corresponds to the inviscid leading eigenvalue (eigenmode) and $\sigma_{1}^{(1)}\left(\phi_{1}^{(1)}\right)$ corresponds to the leading order modification of the leading eigenvalue (eigenmode) due to perturbation by the viscous operator, gives

$$
\sigma_{1}^{(1)}=\frac{\left\langle\phi_{1}^{(0)+} \mid L^{(1)} \phi_{1}^{(0)}\right\rangle}{\left\langle\phi_{1}^{(0)+} \mid \phi_{1}^{(0)}\right\rangle}=\frac{\left\langle\phi_{1}^{(0)+} \mid \Delta_{2 D} \phi_{1}^{(0)}\right\rangle}{\left\langle\phi_{1}^{(0)+} \mid \phi_{1}^{(0)}\right\rangle}-k_{y}^{2},
$$

where $\phi_{1}^{(0)+}$ is the adjoint eigenmode. The estimate of the viscous damping of the elliptic instability, for a Reynolds number based on the circulation $\operatorname{Re}_{\Gamma}=2000$ has been computed using Eq. (C1). The growth rate nondimensionalized by the strain $2 \pi b^{2} / \Gamma$ is given by

$$
\sigma_{1} \frac{2 \pi b^{2}}{\Gamma}=\sigma_{1}^{(0)} \frac{2 \pi b^{2}}{\Gamma}-\frac{2 \pi}{\operatorname{Re}_{\Gamma}}\left(\frac{b}{a}\right)^{2}\left[\left(k_{y} a\right)^{2}-C_{k_{y m}} a^{2}\right],
$$

where $\sigma_{1}^{(0)} 2 \pi b^{2} / \Gamma$ is the inviscid prediction of Le Dizès and Laporte $^{10}$ and the constant $C_{k_{y m}}$ 


$$
C_{k_{y m}}=\frac{\left\langle\phi_{1 m}^{(0)+} \mid \Delta_{2 D} \phi_{1 m}^{(0)}\right\rangle}{\left\langle\phi_{1 m}^{(0)+} \mid \phi_{1 m}^{(0)}\right\rangle}=-\frac{6.71}{a^{2}},
$$

has been computed for $k_{y m} a=2.26$, the maximum of the first elliptic instability band using the direct eigenmode $\phi_{1 m}^{(0)}$ and the adjoint eigenmode $\phi_{1 m}^{(0)+}$ obtained for $\operatorname{Re}_{\Gamma}=10^{5}$ (which is close enough to the inviscid limit). This estimate predicts $\sigma_{1} 2 \pi b^{2} / \Gamma=0.5$ for $\operatorname{Re}_{\Gamma}=2000$ for the antisymmetric mode, which is equal to the growth rate of the antisymmetric mode $\sigma_{r} 2 \pi b^{2} / \Gamma=0.5$ obtained in Sec. II D 2. Prediction (C2), plotted in Fig. 2(b), is in very good agreement with the results of the linear stability analysis.

${ }^{1}$ S. C. Crow, "Stability theory for a pair of trailing vortices," AIAA J. 8, 2172 (1970).

${ }^{2}$ D. W. Moore and P. G. Saffman, "The instability of a straight filament in a strain field," Proc. R. Soc. London, Ser. A 346, 413 (1975).

${ }^{3}$ C.-Y. Tsai and S. E. Widnall, "The stability of short waves on a straight vortex filament in a weak externally imposed strain field," J. Fluid Mech. 73, 721 (1976).

${ }^{4}$ R. T. Pierrehumbert, "Universal short-wave instability of two-dimensional eddies in an inviscid fluid," Phys. Rev. Lett. 57, 2157 (1986).

B. J. Bayly, "Three-dimensional instability of elliptical flow," Phys. Rev. Lett. 57, 2160 (1986).

${ }^{6} \mathrm{~F}$. Waleffe, "On the three dimensional instability of strained vortices," Phys. Fluids A 2, 76 (1990).

${ }^{7}$ D. Sipp and L. Jacquin, "Widnall instabilities in vortex pairs," Phys. Fluids 15, 1861 (2003).

${ }^{8}$ P. Billant, P. Brancher, and J.-M. Chomaz, "Three-dimensional stability of a vortex pair," Phys. Fluids 11, 2069 (1999).

${ }^{9}$ F. Laporte and A. Corjon, "Direct numerical simulation on the elliptic instability of a vortex pair," Phys. Fluids 12, 1016 (2000).

${ }^{10}$ S. Le Dizès and F. Laporte, "Theoretical predictions for the elliptical instability in a two-vortex flow," J. Fluid Mech. 471, 120 (2002).

${ }^{11}$ R. R. Kerswell, "Elliptical instability," Annu. Rev. Fluid Mech. 34, 83 (2002).

${ }^{12}$ C. Eloy and S. Le Dizès, "Stability of the Rankine vortex in a multipolar strain field," Phys. Fluids 13, 660 (2001).

${ }^{13}$ E. W. Mayer and K. G. Powell, "Viscous and inviscid instabilities of a trailing vortex," J. Fluid Mech. 245, 91 (1992).

${ }^{14}$ L. Lacaze, K. Ryan, and S. Le Dizès, "Elliptic instability in a strained Batchelor vortex," J. Fluid Mech. 577, 341 (2007).

${ }^{15}$ L. Coquart, D. Sipp, and L. Jacquin, "Mixing induced by Rayleigh-Taylor instability in a vortex," Phys. Fluids 17, 021703 (2005).

${ }^{16}$ S. Leibovich and K. Stewartson, "A sufficient condition for the instability of columnar vortices," J. Fluid Mech. 126, 335 (1983).

${ }^{17}$ G. Ben-Dov, V. Levinski, and J. Cohen, "Optimal disturbances in swirling flows," AIAA J. 42, 1841 (2004).

${ }^{18}$ C. J. Heaton and N. Peake, "Transient growth in vortices with axial flow,"
J. Fluid Mech. 587, 271 (2007).

${ }^{19}$ T. Leweke and C. Williamson, "Cooperative elliptic instability of a vortex pair," J. Fluid Mech. 360, 85 (1998).

${ }^{20} \mathrm{P}$. Luchini, "Reynolds-number-independent instability of the boundary layer over a flat surface: Optimal perturbations," J. Fluid Mech. 404, 289 (2000).

${ }^{21}$ A. Antkowiak and P. Brancher, "Transient growth for the Lamb-Oseen vortex," Phys. Fluids 16, L1 (2004).

${ }^{22}$ D. S. Pradeep and F. Hussain, "Transient growth of perturbations in a vortex column," J. Fluid Mech. 550, 251 (2006).

${ }^{23}$ V. Brion, D. Sipp, and L. Jacquin, "Optimal amplification of the Crow instability," Phys. Fluids 19, 111703 (2007).

${ }^{24}$ D. Sipp, L. Jacquin, and C. Cossu, "Self-adaptation and viscous selection in concentrated 2D vortex dipole," Phys. Fluids 12, 245 (2000).

${ }^{25}$ G. K. Batchelor, An Introduction to Fluid Dynamics (Cambridge University Press, Cambridge, England, 1967).

${ }^{26}$ I. Delbende, J.-M. Chomaz, and P. Huerre, "Absolute/convective instabilities in the bachelor vortex: A numerical study of the linear impulse response," J. Fluid Mech. 355, 229 (1998).

${ }^{27}$ W. S. Edwards, L. S. Tuckerman, R. A. Friesner, and D. C. Sorensen, "Krylov methods for the incompressible Navier-Stokes equations," J. Comput. Phys. 110, 82 (1994).

${ }^{28}$ S. Julien, S. Ortiz, and J.-M. Chomaz, "Secondary instability mechanisms in the wake of a flat plate," Eur. J. Mech. B/Fluids 23, 157 (2004).

${ }^{29}$ S. E. Widnall, "Structure and dynamics of vortex filaments," Annu. Rev. Fluid Mech. 7, 141 (1975).

${ }^{30}$ The misprints in formulas (6.1) and (6.2) in Le Dizès and Laporte (Ref. 10) have been corrected by replacing $b^{2} / a_{i}^{2}$ by $b^{4} / a_{i}^{4}$ when appropriate.

${ }^{31}$ M. J. Landman and P. G. Saffman, "The three-dimensional instability of strained vortices in a viscous fluid," Phys. Fluids 30, 2339 (1987).

${ }^{32}$ P. G. Saffman, Vortex Dynamics (Cambridge University Press, Cambridge, England, 1992).

${ }^{33}$ P. J. Schmid and D. S. Henningson, Stability and Transition in Shear Flows (Springer-Verlag, New York, 2001).

${ }^{34} \mathrm{P}$. Corbett and A. Bottaro, "Optimal perturbations for boundary layers subject to stream-wise pressure gradient," Phys. Fluids 12, 120 (2000).

${ }^{35}$ E. L. Ince, Ordinary Differential Equations (Dover, New York, 1944).

${ }^{36}$ D. C. Hill, "Adjoint systems and their role in the receptivity problem for boundary layers," J. Fluid Mech. 292, 183 (1995).

${ }^{37}$ H. Salwen and C. E. Grosch, "The continuous spectrum of the OrrSommerfeld equation. Part 2. Eigenfunction expansions," J. Fluid Mech. 104, 445 (1981).

${ }^{38}$ C. P. Caulfield and R. R. Kerswell, "The nonlinear development of threedimensional disturbances at hyperbolic stagnation points: A model of the braid region in mixing layers," Phys. Fluids 12, 1032 (2000).

${ }^{39} \mathrm{P}$. Andersson, M. Berggren, and D. Henningson, "Optimal disturbances and bypass transition in boundary layers," Phys. Fluids 11, 134 (1999).

${ }^{40} \mathrm{~S}$. Leblanc and C. Cambon, "On the three-dimensional instabilities of plane flows subjected to Coriolis force," Phys. Fluids 9, 1307 (1997).

${ }^{41}$ D. Fabre, D. Sipp, and L. Jacquin, "Kelvin waves and the singular modes of the Lamb-Oseen vortex," J. Fluid Mech. 551, 235 (2006). 